\title{
Gluon-photon signatures for color octet at the LHC (and beyond)
}

\author{
G. Cacciapaglia, ${ }^{a}$ A. Deandrea, ${ }^{a}$ T. Flacke ${ }^{b}$ and A.M. Iyer ${ }^{a}$ \\ ${ }^{a}$ Univ. Lyon, Université Claude Bernard Lyon 1, CNRS/IN2P3, UMR5822 IP2I, \\ F-69622, Villeurbanne, France \\ ${ }^{b}$ Center for Theoretical Physics of the Universe, Institute for Basic Science (IBS), \\ Daejeon 34126, Korea \\ E-mail: g.cacciapaglia@ipnl.in2p3.fr, deandrea@ipnl.in2p3.fr, \\ flacke@ibs.re.kr, a.iyer@ipnl.in2p3.fr
}

ABSTRACT: We consider a color octet scalar particle and its exotic decay in the channel gluon- $\gamma$ using an effective Lagrangian description for its strong and electromagnetic interactions. Such a state is present in many extensions of the Standard Model, and in particular in composite Higgs models with top partial compositeness, where couplings to photons arise via the Wess-Zumino-Witten term. We find that final states with one or two photons allow for a better reach at the LHC, even for small branching ratios. Masses up to $1.2 \mathrm{TeV}$ can be probed at the HL-LHC by use of all final states. Finally, we estimate the sensitivity of the hadronic FCC.

KeYwords: Exotics, Hadron-Hadron scattering (experiments), Beyond Standard Model, Jets, Photon production

ARXIV EPRINT: 2002.01474 


\section{Contents}

1 Introduction 1

2 Current bounds on color octet single and pair production 3

2.1 On the relevance of the photon 6

$\begin{array}{ll}2.2 & \text { The bearable smallness of } C_{t} \text { in composite models }\end{array}$

3 Photons in color octet pair production: collider strategy 9

$\begin{array}{lll}3.1 & \text { Background estimation } & 10\end{array}$

$\begin{array}{lll}3.2 & \text { Signal and background acceptances } & 13\end{array}$

$\begin{array}{lll}3.3 & \text { Signal sensitivities } & 15\end{array}$

$\begin{array}{ll}3.4 & \text { The next collider: FCC-hh } \\ \end{array}$

$\begin{array}{llr}4 \text { Conclusion } & 20\end{array}$

$\begin{array}{ll}\text { A Bookkeeping of neutral color octet models } & 20\end{array}$

B Current limits from single production $\quad 22$

\section{Introduction}

Color octet particles are present in various extensions of the Standard Model (SM), ranging from supersymmetric models to composite models for the electroweak sector. Examples include gluinos in supersymmetry, top-gluons in strong electroweak sectors, and KaluzaKlein particles. Color octet properties have been widely discussed in the literature, with, in recent years, particular focus on the LHC physics, see for example [1] and references therein. This is justified by the huge potential for exclusion or discovery which the present or future options for the LHC offer in this specific sector. In the following we shall focus on a particular class of color octet particles, those that are scalars or pseudo-scalars $(\Phi)$, as they have a specific relevance for composite models for the electroweak sector [2]: composite color octets are typically made of fundamental fermions of an underlying strong dynamics associated to top partial compositeness [3, 4].

Yet, the properties and strategies to determine bounds and future prospects for discovery do not depend crucially on the specific model the color octet stems from. In fact, the couplings of the new state to SM particles are mainly dictated by their gauge quantum numbers. First, thanks to QCD gauge interactions, the color octet scalar and pseudo-scalar can be pair-produced at hadron colliders in a model independent way, with cross sections that only depend on the mass. Single couplings to a pair of quarks, with typical preference for tops, are also allowed producing decays into a pair of jets or $t \bar{t}$. Finally, loops of tops 
generate in turn couplings to a pair of gluons, to a gluon and a photon and to a gluon and a $Z$ boson. In composite models, the loop induced couplings also receive a contribution from topological terms, i.e. the Wess-Zumino-Witten (WZW) term. The coupling to gluons, and in minor extent the one to light quarks, also allows for single production. In composite scenarios, the WZW interactions are of particular interest as they carry information about the details of the microscopic properties of the underlying dynamics, while the composite scalar and pseudo-scalar may be among the lightest states of the theory if they arise as pseudo-Nambu-Goldstone bosons (pNGBs). A general analysis of jet-photon and jet- $Z$ resonances at the LHC has been presented in [5].

In this work, we will reconsider the phenomenology of a color octet scalar and pseudoscalar by focusing on specific composite scenarios with top partial compositeness. In fact, a common feature of models formulated in terms of a fermionic strongly coupled gauge theory [4] is the presence of specific additional (light) spin- 0 resonances, namely two neutral singlets and a color octet pseudo-scalar $[2,6]$. In these models, the decay rate in the gluon$\gamma$ channel can be predicted and turns out to be sizeable. Focusing on this channel $[7,8]$ is, therefore, particularly well motivated. We compare the gluon-gluon decay mode to the gluon- $\gamma$ one in the LHC setup. It is interesting to note that already in the 1980's these channels were compared at TeVatron [7] for their potential in the search of a strongly interacting electroweak sector. We discuss the implication and the potential of these modes for the color octet $\Phi$ at the LHC and its future high luminosity (HL-LHC) and high energy (HE-LHC) options, as well as future projects (FCC-hh). This will allow to define the detailed analysis strategies for the experimental searches at the LHC, and at future options, of these kinds of resonances. Our work is of particular interest in view of testing models with a strong electroweak sector.

In order to discuss in a general way the color octet interactions across different models, we shall consider effective interactions encoded in the effective Lagrangian for a pseudoscalar octet discussed in [6], which contains general features present in typical extensions of the SM. In particular, the color octet decays into $t \bar{t}, g g, g \gamma$, and $g Z$ are parameterized as follows:

$$
\begin{aligned}
\mathcal{L}_{\Phi}= & \frac{1}{2}\left(D_{\mu} \Phi^{a}\right)^{2}-\frac{1}{2} M_{\Phi}^{2}\left(\Phi^{a}\right)^{2}+i C_{t} \frac{m_{t}}{f_{\Phi}} \Phi^{a} \bar{t} \gamma_{5} \frac{\lambda^{a}}{2} t \\
& +\frac{\alpha_{s} \kappa_{g}}{8 \pi f_{\Phi}} \Phi^{a} \epsilon^{\mu \nu \rho \sigma}\left[\frac{1}{2} d^{a b c} G_{\mu \nu}^{b} G_{\rho \sigma}^{c}+\frac{e \kappa_{\gamma}}{g_{s} \kappa_{g}} G_{\mu \nu}^{a} F_{\rho \sigma}-\frac{e \tan \theta_{W} \kappa_{Z}}{g_{s} \kappa_{g}} G_{\mu \nu}^{a} Z_{\rho \sigma}\right],
\end{aligned}
$$

where $f_{\Phi}$ is a mass scale (corresponding to the decay constant of the composite $\Phi$ ), while the covariant derivative contains QCD interactions with gluons. The relative value of the photon coupling, $\kappa_{\gamma}$, and the $Z$ coupling, $\kappa_{Z}$, depend on the electroweak quantum numbers of the multiplet $\Phi$ belongs to. In the following, for simplicity, we will focus on a weak isosinglet, for which

$$
\kappa_{\gamma}=\kappa_{Z} \equiv \kappa_{B}
$$

as this case applies directly to composite Higgs examples. As a bookkeeping, we present other cases in appendix A. In the underlying models considered in [6], the color octet arises as a bound state of color triplet fermions $\chi$ with hypercharge $Y_{\chi}=1 / 3$ or $2 / 3$, thus the 


\begin{tabular}{|c|c|c|}
\hline & $\frac{\mathrm{BR}(\Phi \rightarrow g \gamma)}{\mathrm{BR}(\Phi \rightarrow g g)}$ & $\frac{\mathrm{BR}(\Phi \rightarrow g Z)}{\mathrm{BR}(\Phi \rightarrow g g)}$ \\
\hline$Y_{\chi}=1 / 3$ & 0.048 & 0.014 \\
$Y_{\chi}=2 / 3$ & 0.19 & 0.058 \\
\hline
\end{tabular}

Table 1. Values of ratios of BRs in di-bosons for the pseudo-scalar octet isosinglet at a mass of $1 \mathrm{TeV}$. The mass fixes the dependence due to the running of the strong gauge coupling, $\alpha_{s}(1 \mathrm{TeV})=$ 0.0881 is used for this evaluation. The $Y_{\chi}=1 / 3(2 / 3)$ will be referred to as the pessimistic (optimistic) case corresponding to its reach in the photon channels, while the decay into $g Z$ is always subleading.

ratio $\kappa_{B} / \kappa_{g}=2 Y_{\chi}$ is also fixed. In turn, this property fixes the relative branching fractions amongst the bosonic final states, as given in table 1. We will use these branching fractions as benchmarks, but results will be presented also for generic $\kappa_{B} / \kappa_{g}$.

The ratio of the partial widths to tops vs. gluons is [6]

$$
\frac{\Gamma_{\Phi \rightarrow t \bar{t}}}{\Gamma_{\Phi \rightarrow g g}}=\frac{48 \pi^{2}}{5 \alpha_{s}^{2}} \frac{C_{t}^{2}}{\kappa_{g}^{2}} \frac{m_{t}^{2}}{M_{\Phi}^{2}}\left(1-4 \frac{m_{t}^{2}}{M_{\Phi}^{2}}\right)^{1 / 2}
$$

thus it scales with the ratio $C_{t}^{2} / \kappa_{g}^{2}$, which we leave as a free parameter. Note that all ratios of branching fractions are independent on the scale $f_{\Phi}$, which is only relevant for the total width of the color octet (and single production rates). In the models we consider, the total width is always very small compared to the mass.

\section{Current bounds on color octet single and pair production}

For QCD pair production of $\Phi$, with subsequent decays into two pairs of $t \bar{t}$ or two pairs of gluons, existing 4-top searches and searches for a pair of di-jet resonances yield bounds on the mass of the color octet that only depend on the branching ratios $\mathrm{BR}(\Phi \rightarrow t \bar{t})$ and $\mathrm{BR}(\Phi \rightarrow g g)$ (for the benchmark composite models, only the ratio $C_{t} / \kappa_{g}$ is relevant, as the relative rates in $g \gamma$ and $g Z$ are fixed). Searches for 4-top final states [9, 10] at the LHC run I were interpreted in a color octet model (sgluon), thus they can be directly applied to our case, while searches for di-jet pairs are not very sensitive to the color structure of the decaying resonances. In figure 1 (left) we show the run I bounds on the cross section for the above-mentioned 4-top searches [9, 10] and for the jet final state [11]. The solid black line shows, for reference, the QCD pair production at $\sqrt{s}=8 \mathrm{TeV}$ at LO in QCD. ${ }^{1}$ At run II, the color octet interpretation for 4-top searches has been dismissed, thus we need to use a recast of the searches, which is only available in ref. [13] for the same-sign lepton search of ref. [14], ${ }^{2}$ which is based on an integrated luminosity of $35.9 \mathrm{fb}^{-1}$. In

\footnotetext{
${ }^{1}$ We calculate the color octet pair production cross section at leading order using MadGraph 5 with the NNPDF23LO (as_0130_qed) PDF set without applying any $K$ - factor. As shown in [12], the NLO $K-$ factor for color octet pair production is close to one for $M_{\Phi}$ at the TeV scale.

${ }^{2}$ The CMS 4-top search in the same-sign lepton channel [13] is based on the $36 \mathrm{fb}^{-1}$ dataset. A CMS search with $137 \mathrm{fb}^{-1}$ became available recently [15]. Further 4-top ATLAS and CMS searches with 36 $\mathrm{fb}^{-1}$ are also available [16-19] but require non-trivial recasting in order to obtain a bound on color octet resonances. We therefore restrict ourselves to [14] for which the recast [13] is available.
} 

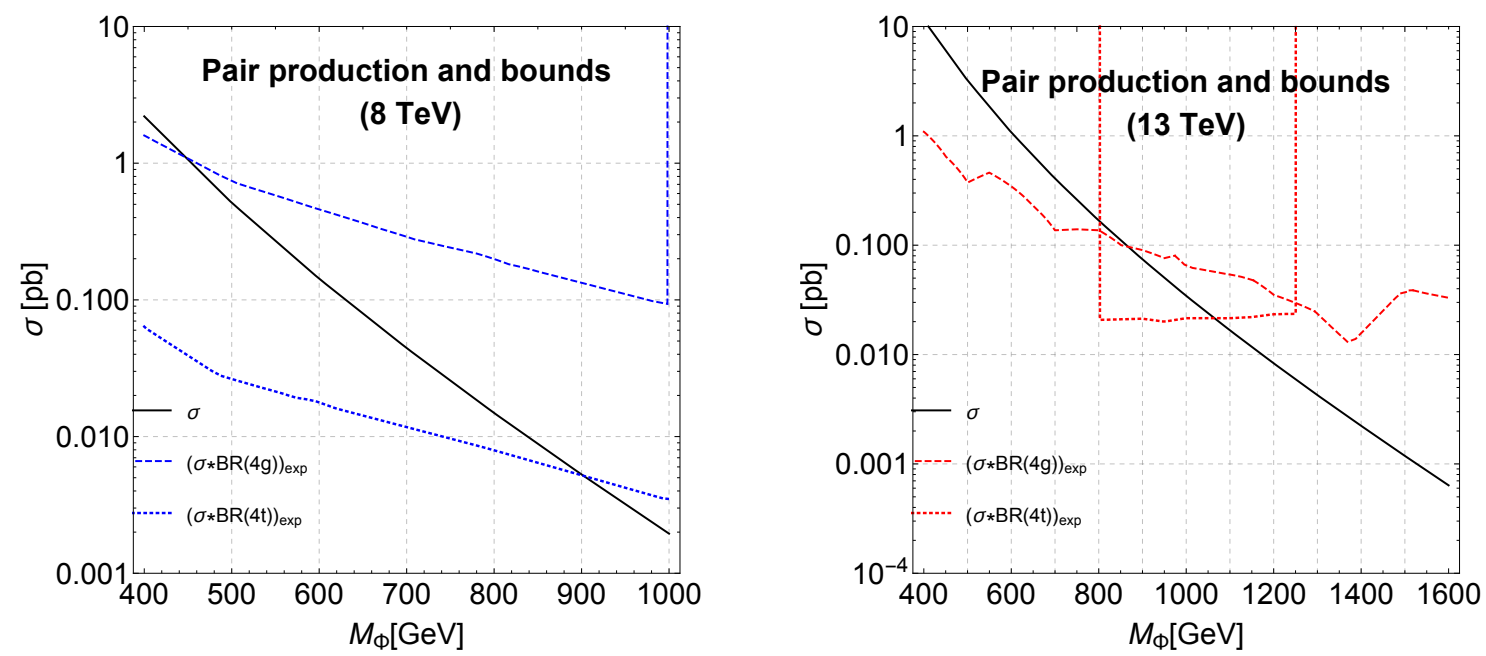

Figure 1. Observed LHC bounds on cross sections from 4-top searches and di-jet-pair searches at run I (left) and run II (right). For reference, the black lines show the total QCD pair production cross section at the respective center-of-mass energy.

figure 1 (right) we show the bound on the cross section, together with the ATLAS and CMS jet searches [20, 21] that are based on $36.7 \mathrm{fb}^{-1}$ and $35.9 \mathrm{fb}^{-1}$ integrated luminosity respectively, together with the $\mathrm{LO} \mathrm{QCD}$ cross section at $\sqrt{s}=13 \mathrm{TeV}$. To translate these bounds into a limit on the color octet mass, it is enough to rescale the total production cross section by the branching ratios, which only depend on ratios of couplings. For the two benchmark models, with reference values $\kappa_{B} / \kappa_{g}=2 / 3$ and $4 / 3$, we show the excluded regions in the $M_{\Phi}$ vs. $C_{t} / \kappa_{g}$ plane in figure 2 . The bounds for $\kappa_{B} / \kappa_{g}=4 / 3$ are marginally weaker because branching fractions into $g \gamma$ (and $g Z$ ) are larger in this case, and events with these decays evade detection in the 4-top and di-jet-pair searches. We see that the bounds on $M_{\Phi}$ range from $\sim 800 \mathrm{GeV}$ in the 4 -jet region to $\sim 1 \mathrm{TeV}$ in the 4-top region, with a 'hole' reaching down to $\sim 600 \mathrm{GeV}$ for intermediate $C_{t} / \kappa_{g} \approx 5 \%$ due to the run II 4 -top search loosing steam because of triggers.

The color octet $\Phi$ can also be singly produced in gluon fusion via its WZW interaction (top loops give a sub-leading contribution in the relevant mass range that is not excluded by pair production). Unlike QCD pair production, the single production cross section depends not only on the octet mass but also on $\kappa_{g} / f_{\Phi} \cdot{ }^{3}$ The different resonant final states are $t \bar{t}, g g$, $g \gamma$, and $g Z$, where the branching fraction between the $t \bar{t}$ and the gauge boson final states is controlled by $C_{t} / \kappa_{g}$, while the ratios between the boson channels with $\gamma / Z$ and purely hadronic are controlled by $\kappa_{B} / \kappa_{g}$ (benchmark values given in table 1 ). These final states are covered by run II resonance searches in $t \bar{t}$ [23-25] with $36 \mathrm{fb}^{-1}$ integrated luminosity, lowmass and high-mass $j j$ searches [26-30] with $36-139 \mathrm{fb}^{-1}$ datasets, and the excited quark searches in $j \gamma[31,32]$ at $\sqrt{s}=13 \mathrm{TeV}$ based on $\sim 36 \mathrm{fb}^{-1}$, while no direct search is available for a $j Z$ resonance (which, however, has a low branching ratio in our focus models). For the $j \gamma$ final state, the $13 \mathrm{TeV}$ searches only apply to invariant masses above $1 \mathrm{TeV}$, therefore

\footnotetext{
${ }^{3}$ In models with BSM QCD bound state color octets, further single-production mechanisms are possible, see e.g. [22].
} 

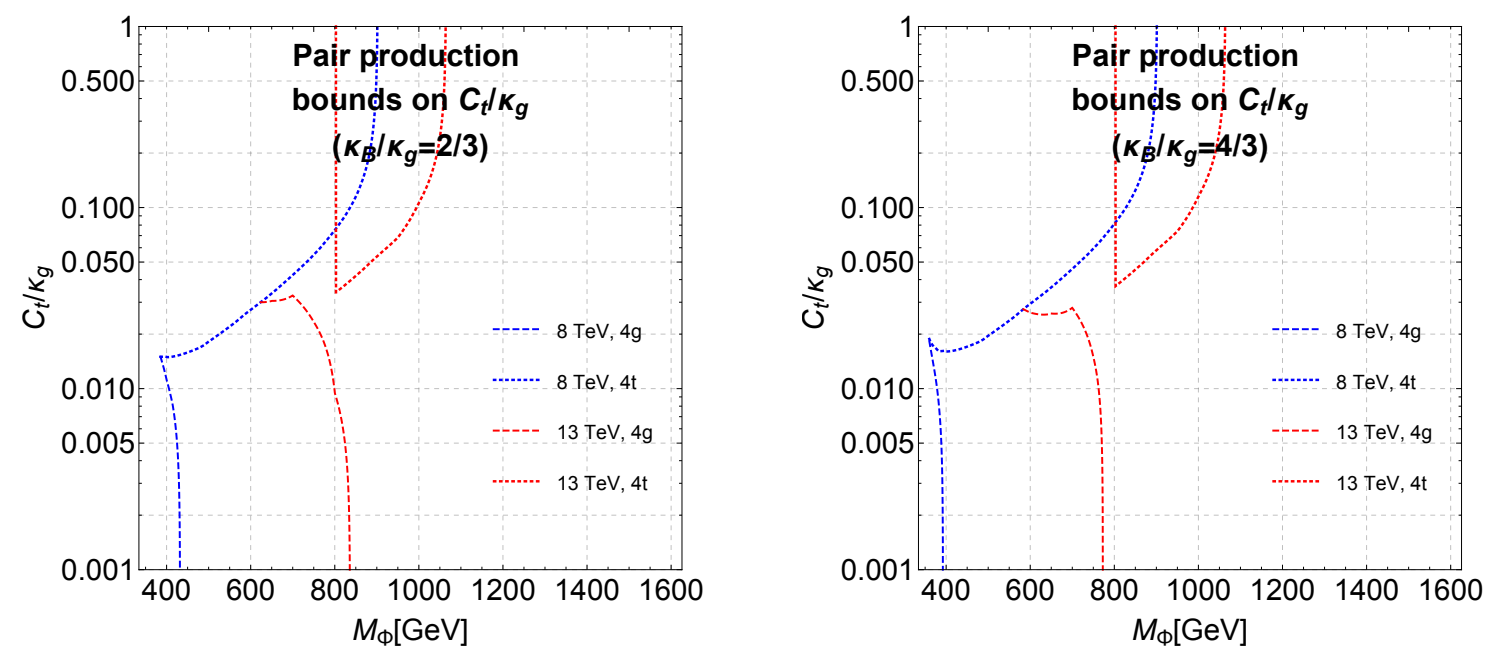

Figure 2. Excluded regions in the $M_{\Phi}$ vs. $C_{t} / \kappa_{g}$ parameter space for the two benchmark models, with branching ratios given in table 1 .
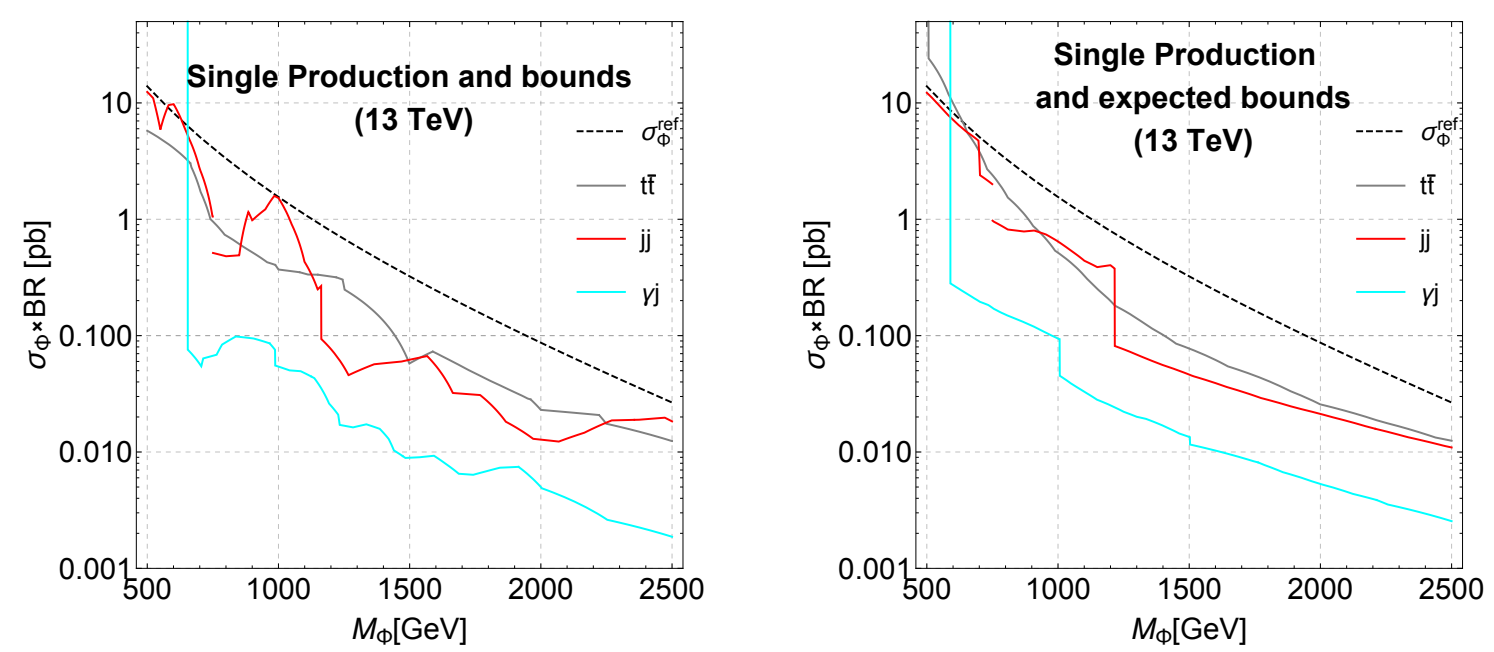

Figure 3. Current observed (left) and expected (right) experimental bounds on the production cross section of $t \bar{t}, j j$, and $j \gamma$ resonances as a function of the resonance mass. As a reference, we give the single production cross section of $\Phi$ for $\kappa_{g} / f_{\Phi}=10 \mathrm{TeV}^{-1}$ as a function of $M_{\Phi}$. The production cross section scales with $\left(\kappa_{g} / f_{\Phi}\right)^{2}$.

we also consider run I searches at $8 \mathrm{TeV}[33,34]$ to cover the lower mass range. In figure 3 (left) we collect the observed bounds on cross section times branching ratio in the various channels, together with the single $\Phi$ cross section for $\kappa_{g} / f_{\Phi}=10 \mathrm{TeV}^{-1}$ for reference. For the $j \gamma$ searches at $8 \mathrm{TeV}$, the observed bound (relevant for $600<M_{\Phi}<1000 \mathrm{GeV}$ ), is plotted rescaled by the ratio of production cross section in the two energy regimes for the color octet, i.e. we plot $\sigma_{\text {limit }}(j \gamma) \times \sigma_{13 \mathrm{TeV}}(g g \rightarrow \Phi) / \sigma_{8} \mathrm{TeV}(g g \rightarrow \Phi)$.

The cross section limits can be directly translated into bounds on the parameter space of the color octet. In particular, for fixed branching ratios, we can extract an upper bound 

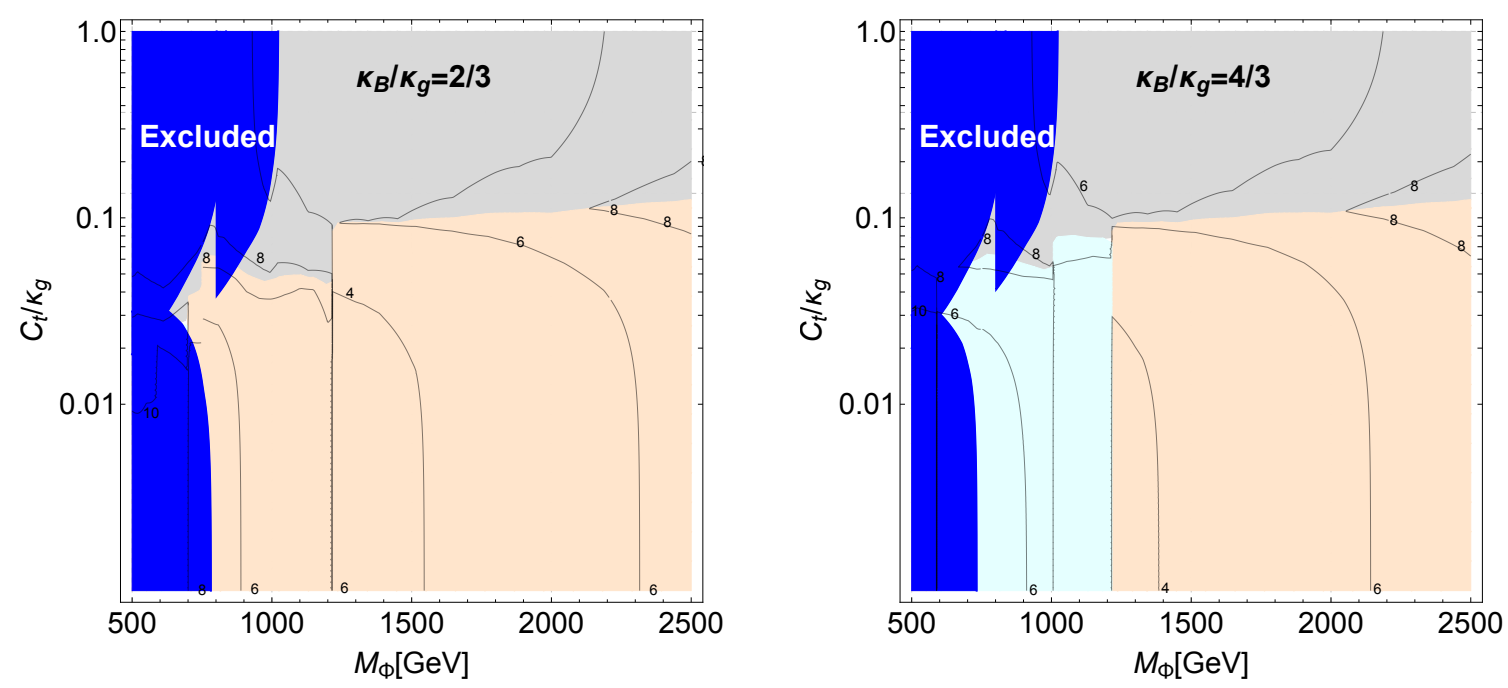

Figure 4. Expected bounds on color octet single production in the $M_{\Phi}$ vs. $C_{t} / \kappa_{g}$ plane for $\kappa_{B} / \kappa_{g}=2 / 3$ and $4 / 3$. The contours show the upper bound on $\kappa_{g} / f_{\Phi}\left(\right.$ in $\left.\mathrm{TeV}^{-1}\right)$. The dark-blue areas are excluded by pair production searches. In the grey areas, the strongest bound arises from $t \bar{t}$ resonance searches. In the orange (cyan) areas, the currently strongest bound arises from di-jet $($ jet- $\gamma)$ searches.

on the couplings to gluon, $\kappa_{g} / f_{\Phi}$, relevant for the rates in single production. Some plots visualizing the observed bounds can be found in appendix B. Here, however, we want to focus on another point: comparing the reach of the di-jet, $t \bar{t}$ and jet- $\gamma$, we would like to highlight in what parameter space the decay model with a photon becomes relevant, notwithstanding the smaller branching ratio. As the observed bounds strongly vary with $M_{\Phi}$ due to statistical fluctuations, see left panel of figure 3 , to obtain more clear indications we used the expected bounds, as shown in the right panel of figure 3 .

\subsection{On the relevance of the photon}

As in general there are too many free parameters, we first focus on the two benchmark models: in figure 4 we show the upper limit on $\left(\kappa_{g} / f_{\Phi}\right)$ in the plane $C_{t} / \kappa_{g}$ vs. $M_{\Phi}$, where the dark blue region is excluded by pair production (C.f. figure 2). As already mentioned, we use the expected bounds in order to obtain more readable figures, where the actual observed bound is numerically close up to statistical fluctuations (see figure 3 and plots in appendix B). The shaded regions indicate which channel provides the strongest limit, with grey corresponding to $t \bar{t}$, orange to $j j$ and cyan to $j \gamma$. The comparison is, however, not completely fair because some $j j$ searches include $139 \mathrm{fb}^{-1}$ integrated luminosity while $t \bar{t}$ and $j \gamma$ are based on only $36 \mathrm{fb}^{-1}$ of data (with the latter also partially based on $8 \mathrm{TeV}$ searches). Thus the grey and cyan regions underestimate the actual potential of these two final states. Nevertheless, the plots clearly show that $t \bar{t}$ dominates as soon as a significant coupling to tops is present, $C_{t} / \kappa_{g} \gtrsim 0.1$.

To show more general results, not only limited to the benchmark models, we now focus on the region where the $t \bar{t}$ final state is negligible and the decays into gauge bosons 


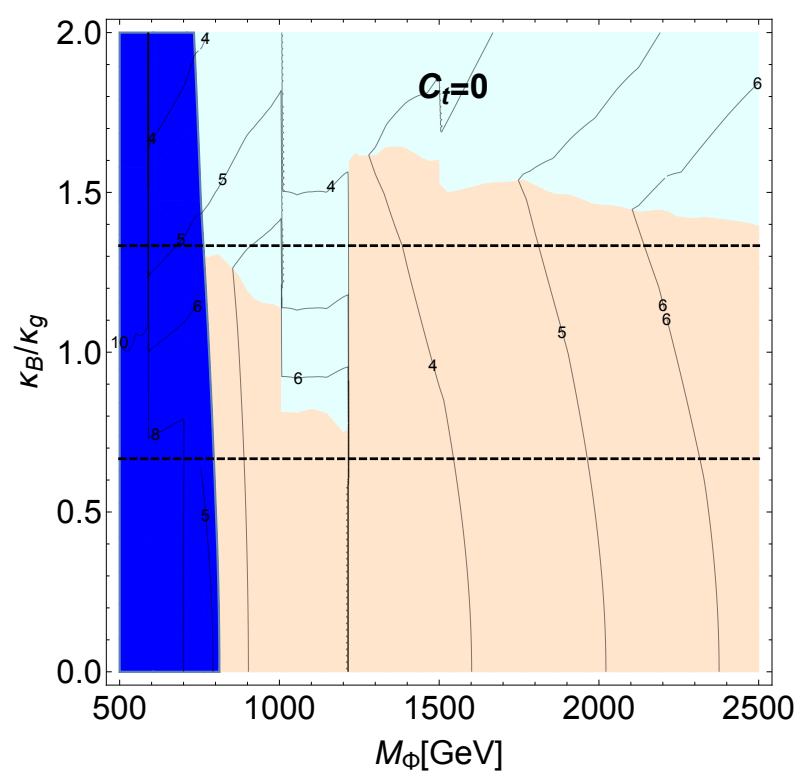

Figure 5. Expected bounds on color octet single production in the $M_{\Phi}$ vs. $\kappa_{B} / \kappa_{g}$ plane. The dark blue area is excluded from pair production bounds, while the orange (cyan) area labels where the dominant bound arises from the $j j(j \gamma)$ searches. Contours show the upper bound on $\kappa_{g} / f_{\Phi}$ in $\mathrm{TeV}^{-1}$. For reference, the horizontal dashed lines indicate fixed $\kappa_{B} / \kappa_{g}$ ratios of $2 / 3$ (lower) and $4 / 3$ (higher).

dominate. A critical discussion of the interest of this assumption in composite Higgs models of reference is presented in the next subsection. In figure 5 we show bounds on $\kappa_{g} / f_{\Phi}$ in the plane $\kappa_{B} / \kappa_{g}$ vs. $M_{\Phi}$ for $C_{t}=0$, with the same conventions for the shaded regions as in figure 4. The horizontal dashed lines correspond to the two benchmark models, showing that the $Y_{\chi}=1 / 3$ bounds are currently dominated by the $j j$ final state (with the caveat on the lower luminosity/energy available in the $j \gamma$ channel), while for $Y_{\chi}=2 / 3$, bounds from the $j j$ and $j \gamma$ final state compete. Inclusion of more data in the $j \gamma$ channel will, however, further extend the relevance of the $j \gamma$ final state compared to the current plot. These results clearly show that the final state with a photon is typically very important in this class of models, and it will be our main focus in the next section.

\subsection{The bearable smallness of $C_{t}$ in composite models}

From the single and pair production bounds we see that the bosonic decay modes (into di-jets or jet-photon) only play a dominant role if $C_{t} / \kappa_{g} \lesssim 0.1$, as otherwise the $4 \mathrm{t}$ and $t \bar{t}$ searches yield best bounds. A priori, the WZW couplings of the color octet $\Phi$ to gauge bosons and its couplings to SM fermions are not directly related, such that small or even vanishing $C_{t} / \kappa_{g} \lesssim 0.1$ is viable. Nevertheless, in models where $\Phi$ arises from a composite sector which is the source of electroweak symmetry breaking and the generation of the top-quark mass, the underlying structure can link WZW and fermion couplings. In this section, we therefore review the relation between top and WZW couplings in composite Higgs models with top partial compositeness in order to assess whether a small $C_{t}$ can be 
naturally obtained. We remark that these models are one of many examples to which our analysis can apply, so they should just be taken as a guide.

The models of top partial compositeness we consider were first proposed in $[3,4]$ : they consist in a confining gauge group $\mathcal{G}_{\mathrm{HC}}$ with two species of fermions, $\psi$ and $\chi$, which transform under different irreducible representations of $\mathcal{G}_{\mathrm{HC}}$. In particular, the $\psi$ 's carry only electroweak quantum numbers and are responsible for generating a composite Higgs upon condensation. The $\chi$ 's carry QCD charges and hypercharge and are introduced to obtain fermionic bound states which serve as vector-like top partners (in the following called "baryons"). The quantum numbers are chosen so that baryons, i.e. spin- $1 / 2$ resonances, are formed out of the two species: depending on the hypercharge $Y_{\chi}=1 / 3$ or $2 / 3$, we have:

$$
\mathcal{B}_{j}=\left\{\begin{array}{l}
\langle\psi \psi \chi\rangle \text { or }\langle\psi \bar{\psi} \bar{\chi}\rangle, \text { for } Y_{\chi}=2 / 3 \\
\langle\psi \chi \chi\rangle \text { or }\langle\bar{\psi} \bar{\chi} \chi\rangle \text { or }\langle\psi \bar{\chi} \bar{\chi}\rangle, \text { for } Y_{\chi}=1 / 3 .
\end{array}\right.
$$

The baryons $\mathcal{B}_{j}$ are the resonances that mix to the SM top fields in order to give them mass via the composite Higgs.

Beyond the top-partners, there are further bound states made up from $\chi$ 's. In particular, analogous to the Higgs from $\psi$ condensation, the $\chi$ 's generate a color octet pNGB $\Phi .{ }^{4}$ The WZW couplings of the color octet bound state $\Phi$ are determined by the quantum numbers of the underlying $\chi$, while the coupling to tops depends on the nature of the baryons $\mathcal{B}_{j}$ coupling to the left and right-handed tops. In ref. [6], it was found that

$$
\frac{C_{t}}{\kappa_{g}}=\frac{n_{\chi}}{d_{\chi}}
$$

where $d_{\chi}$ is the dimension of the underlying $\chi$ fermion under the confining gauge group, while $n_{\chi}=n_{\chi L}+n_{\chi R}$ is an integer, where $n_{\chi L / R}$ count how many $\chi-\bar{\chi}$ fermions appear in the baryons mixing to left and right-handed tops respectively. From eq. (2.1), we see that this depends on the hypercharge of $\chi$. For $Y_{\chi}=2 / 3, n_{L / R}= \pm 1$, and thus $n_{\chi}= \pm 2,0$. For $Y_{\chi}=1 / 3, n_{L / R}= \pm 2,0$, and thus $n_{\chi}= \pm 4, \pm 2,0$. In the case $n_{\chi}=0$, therefore, vanishing couplings to the top can be obtained. Furthermore, $C_{t} \ll \kappa_{g}$ could also be realised in models where $d_{\chi}$ is sufficiently large. In table 2 we list values of $d_{\chi}$ for the 12 minimal models of top partial compositeness of $[4,6,35]$. We see that while for instance M2 and M7 have moderately large $d_{\chi}$, achieving $C_{t} / \kappa_{g}<0.1$ is only possible within the minimal models with $n_{\chi}=0$.

The above results, however, were based on a spurion analysis where effective operators are constructed in terms of the spurions describing the partial compositeness mixing of the left-handed and right-handed tops. If the top partners are light compared to the other resonances, then the dominant contribution to the top mass may come from the direct mixing with the lightest baryon resonance. It was found in [36] that the two approaches based on operator analysis and on the baryon mixing are not completely equivalent: the

\footnotetext{
${ }^{4}$ The composite Higgs (by construction) and the color octet are present in all models of top partial compositeness. The models also contain further neutral, electroweakly charged, and colored pNGBs, which depend on the number and representations of $\chi$ 's and $\psi$ 's under $\mathcal{G}_{\mathrm{HC}}($ see $[4,6,35])$.
} 


\begin{tabular}{|l|c|c|c|c|c|c|}
\hline & $\mathrm{M} 1$ & $\mathrm{M} 2$ & $\mathrm{M} 3$ & $\mathrm{M} 4$ & $\mathrm{M} 5$ & $\mathrm{M} 6$ \\
\hline $\mathcal{G}_{\mathrm{HC}}$ & $\mathrm{SO}(7)$ & $\mathrm{SO}(9)$ & $\mathrm{SO}(7)$ & $\mathrm{SO}(9)$ & $\mathrm{Sp}(4)$ & $\mathrm{SU}(4)$ \\
\hline$Y_{\chi}$ & $1 / 3$ & $1 / 3$ & $2 / 3$ & $2 / 3$ & $1 / 3$ & $1 / 3$ \\
$d_{\chi}$ & 8 & 16 & 7 & 9 & 4 & 4 \\
\hline & $\mathrm{M} 7$ & $\mathrm{M} 8$ & $\mathrm{M} 9$ & $\mathrm{M} 10$ & $\mathrm{M} 11$ & $\mathrm{M} 12$ \\
\hline $\mathcal{G}_{\mathrm{HC}}$ & $\mathrm{SO}(10)$ & $\mathrm{Sp}(4)$ & $\mathrm{SO}(11)$ & $\mathrm{SO}(10)$ & $\mathrm{SU}(4)$ & $\mathrm{SU}(5)$ \\
\hline$Y_{\chi}$ & $1 / 3$ & $2 / 3$ & $2 / 3$ & $2 / 3$ & $2 / 3$ & $2 / 3$ \\
$d_{\chi}$ & 16 & 5 & 11 & 10 & 6 & 10 \\
\hline
\end{tabular}

Table 2. Values of $d_{\chi}$ for the 12 models based on [4] (see $[6,35]$ for the model list assignments).

couplings of a singlet pseudo-scalar were found to differ qualitatively and numerically. The main origin of this discrepancy traces back to the fact that couplings to the light scalar only arise via the mixing terms, thus diagonalising the mass matrix is not equivalent to diagonalising the couplings. Repeating the calculation in [36] for the case of composite color octet couplings from the mixing with light top partners, the ratio $C_{t} / \kappa_{g}$ is obtained as

$$
\frac{C_{t}}{\kappa_{g}}=\frac{n_{\chi L} \sin ^{2} \alpha_{L}+n_{\chi R} \sin ^{2} \alpha_{R}}{d_{\chi}},
$$

where $\alpha_{L / R}$ are the mixing angles of the left- and right-handed tops to the composite states. Unlike in (2.2), where $n_{\chi L}=-n_{\chi R}$ yields full cancellation and thus a vanishing top coupling, (2.3) implies that perfect cancellation becomes unlikely due to the dependence on the mixing angles, as typically $\sin \alpha_{R}>\sin \alpha_{L}$ to avoid bounds on the left-handed bottom. Thus, if the dominant contribution to the top mass may come from the direct mixing with the lightest baryon resonance, very small $C_{t} / \kappa_{g}$ is only achieved in models with $n_{\chi L}=n_{\chi R}=0$ for $Y_{\chi}=1 / 3$.

This analysis shows that a small $C_{t}$ is not common in composite models, especially if the top mass comes from mixing to light top partners, but example models with naturally small $C_{t}$ exist. We also recall that the relation between top-coupling and the WZW term originates from a combination of demands: a composite Higgs, partial top compositeness and the color octet being the pNGB of the color charged confined sector. The relation of the coupling to $g g$ and $\gamma g$ is more direct and only depends on the value of $Y_{\chi}$, as shown in table 1 .

\section{Photons in color octet pair production: collider strategy}

As we have seen in the previous section, photons from the color octet decays can play a very relevant role in the phenomenology, and searches in single production can be reinterpreted in this framework. However, no pair-production search based on photons exists so far. In this section we will cover this gap and establish a strategy to set up this kind of searches at the LHC (including the HL-LHC run) and at future higher-energy hadron colliders (FCC-hh). 
In this pursuit, we are particularly interested in scenarios where the $\Phi \rightarrow g g$ is the dominant decay mode, thus we will set $C_{t}=0(\operatorname{BR}(\Phi \rightarrow \bar{t} t)=0)$ in the following. While searches exist in the multi-jet final state for pair produced scalars, it must be noted that they are beset by a large irreducible QCD background. We will, therefore, consider final states with one or two photons and compare the sensitivity in these channels to the multi-jet one. To facilitate the comparison, we define ratios of signal significance as follows

$$
\delta_{g g g \gamma} \equiv \frac{Z_{g g g \gamma}}{Z_{g g g g}}=\frac{S_{g g g \gamma} / \sqrt{B_{j j j a}}}{S_{g g g g} / \sqrt{B_{j j j j}}}, \quad \delta_{g g \gamma \gamma} \equiv \frac{Z_{g g \gamma \gamma}}{Z_{g g g g}}=\frac{S_{g g \gamma \gamma} / \sqrt{B_{j j a a}}}{S_{g g g g} / \sqrt{B_{j j j j}}},
$$

where the sensitivities $Z_{x}$ are simplistically defined as ratios of the number of signal events $S_{x}$ divided by the square root of the background events $B_{x}$. Note that we use $g$ and $\gamma$ to indicate parton level gluon and photon in the signal, while we use $j$ and $a$ to indicate detector reconstructed jet and photon in the background to take into account fakes. We will use this ratio as an indicator of the relevance or dominance of the photon final states over the purely hadronic mode: it is evident in the simplistic definition of significance that the ratios are proportional to ratios if branching ratios. In the latter part of this section we will eventually adapt a more generalized version for the estimation of signal sensitivity, as we will discuss later. The analysis developed can be extended to any model that has a $g \gamma$ decay mode, thus we will give results for the benchmark models in table 1, as well as for general values of $B R(\Phi \rightarrow g \gamma) / B R(\Phi \rightarrow g g)$.

The first step in this direction corresponds to determining the backgrounds and the corresponding fake rates for the jjja and jjaa final states. The fake rate is due to the multi-jet background where a photon is radiated by the quark fragmentation or a jet is mistagged as a photon. Given the large production cross sections for the multi-jet processes, these fake rates must be understood to a fair degree of accuracy. We begin with a detailed study of the different backgrounds relevant for our analysis.

\subsection{Background estimation}

To correctly estimate the backgrounds, it is crucial to estimate the expected fake rates in the signal regions. Since the multi-jet scenario is relatively complicated, as a proof of concept we first consider the purity of a $p p \rightarrow j a$ sample. To do so, we generated two samples of events:

$$
\text { 1) } p p>j \gamma(\text { pure sample); } \quad \text { 2) } p p>j j \text { (fake sample); }
$$

where $j=g, q$. We simulate 150000 events at MonteCarlo level for both samples at $14 \mathrm{TeV}$ center of mass, requiring that the total $p_{T}$ of the two outgoing particles must be at least $300 \mathrm{GeV}$. The events are generated using PYTHIA 8 [37], while we use DELPHES 3 [38] for the detector simulation. To include the effect of a jet mistagged as a photon, we use a flat probability $\epsilon=10^{-4}$ in the JetFakeParticle module of DELPHES. Further, we assume the corresponding rate for the jets faking as either an electron or a muon to be zero. The signal selection includes events with at least a single jet and a single isolated photon. Figure 6 gives the purity fraction, defined as $N_{j \gamma} /\left(N_{j \gamma}+N_{j j}\right)$, as a function of the lower $p_{T}$ threshold 


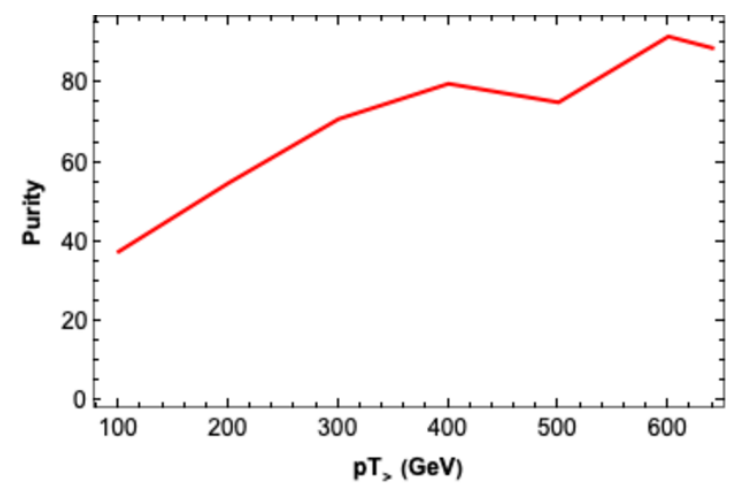

Figure 6. Purity $N_{j \gamma} /\left(N_{j \gamma}+N_{j j}\right)$ of the $j a$ samples with a single photon and a jet.

for the photon (denoted as $p_{T_{>}}$): all events with $p_{T}>p_{T_{>}}$contribute and are normalized to the corresponding cross section. As expected, at lower thresholds we see a lower purity due to larger pollution from the $j j$ events. This is drastically reduced for increasing $p_{T>}$ for the photon, while the upper value owes to the absence of high- $p_{T}$ photons in the samples. The purity for the $j a$ sample increases rapidly with the photon transverse momentum as the jet and the photon from the $p p>j \gamma$ process is one QCD order lower than the one arising due to $p p>j j$. As a validation, we can compare this behavior to the purity in the CMS analysis at $8 \mathrm{TeV}$ [39], which shows good agreement. The absence of pile-up both in the data and in our simulations makes it a reasonable comparison. ${ }^{5}$

Having validated our method, we now consider the purity of the $p p>j j j a$ background. Events with a "real" photon primarily correspond to the radiation of a quark, therefore they are rather similar to events from the pure multi-jet sample. As a result they are at the same QCD and QED order. Since we will consider the pair production of resonances of at-least $1 \mathrm{TeV}$ mass, we limit the matrix element generation of the background events to the following kinematical regions:

- Multi-jet: the scalar $p_{T}$ sum of the outgoing partons is required to be at least $1500 \mathrm{GeV}$;

- $p p>j j j a$ The scalar $p_{T}$ sum of the outgoing partons is required to be at least $1400 \mathrm{GeV}$, while the photon is required to have a $p_{T}$ of at-least $100 \mathrm{GeV}$.

As emphasised earlier, since the $j j j j$ and the $j j j \gamma$ samples are characterized by Feynman diagrams at the same order, the purity in this instance is mainly a measure of the percentage of the jet faking a photon as well as possibility of a hard radiated photon being isolated from the multi-jet sample. With increasing photon $p_{T}$, the probability of a hard radiated photon from the $j j j j$ sample decreases. The left plot of figure 7 illustrates the purity for the $j j j a$ sample in our simulation. We accept events with at-least three jets and an isolated photon from both multi-jet and $j j j \gamma$ samples. Owing to the similarity between the two, the multi-jet sample dominates for low and intermediate $p_{T}$ on account of its greater

\footnotetext{
${ }^{5}$ The corresponding distribution is not available at $13 \mathrm{TeV}$.
} 

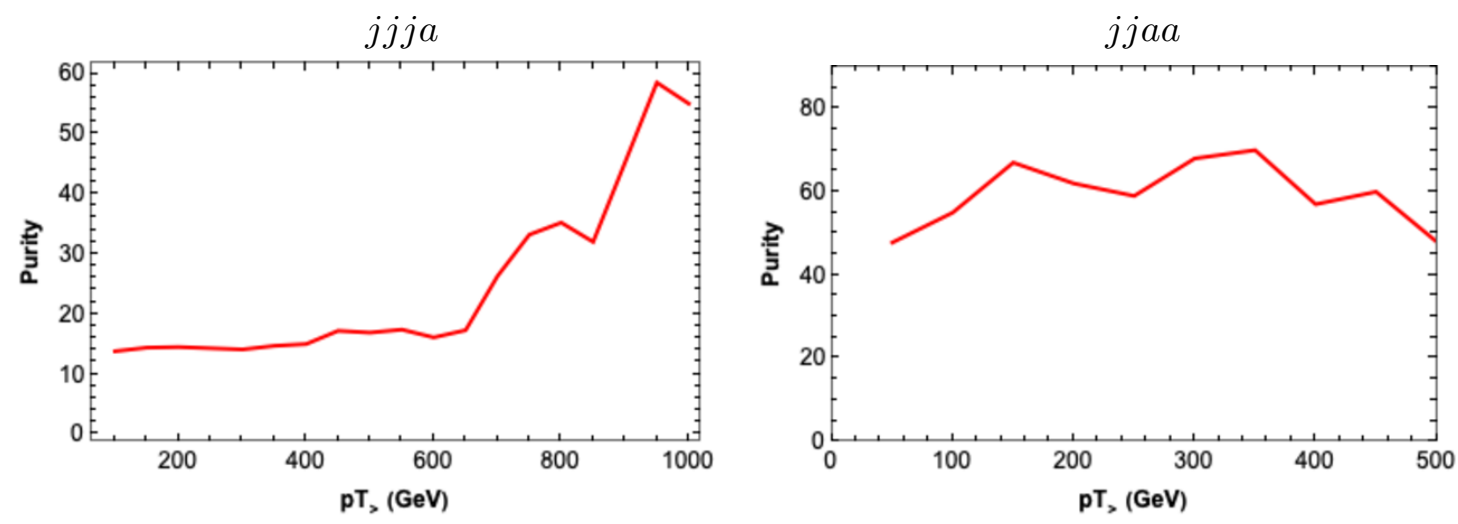

Figure 7. Purity of the jjja (left) and jjaa (right) samples. In the former, pollution comes from $j j j j$ events; for the latter, pollution derives from $j j j \gamma$ events.

cross-section, thus the multi-jet background will dominate for low mass resonances. With increasing $p_{T}$, one observes an increase in the purity due to the fact that the photon from the $j j j \gamma$ samples has a mildly larger tail than the one due to $j j j j$ (the latter sensitive to the "softer" Bremsstrahlung). The fluctuations at the tail of the curve can be attributed to a lack of statistics. Our analysis shows, therefore, that the multi-jet background is relevant for the $g g g \gamma$ signal and the results of our later analysis must be taken with caution: in fact, only data driven techniques allow for a reliable estimate of this background, while our method is limited by statistics and the accuracy of the MonteCarlo in characterizing the tails of the event distributions.

Finally, to account for the possibility of the color octet pair decaying in two photons and two gluons, we estimate the corresponding jjaa background. In this case, the "pure" background is due to $p p>j j \gamma \gamma$, with contamination from the $p p>j j j \gamma$ processes. The background events $j j \gamma \gamma$ are similar to the di-jet events considered earlier with the additional radiation of photons off the quarks. To match the kinematics of the signal, we further require that the photons have $p_{T}>150 \mathrm{GeV}$ at particle level, while the scalar $p_{T}$ sum of the outgoing partons is required to be at-least $500 \mathrm{GeV}$. The fakes are mainly due to $j j j \gamma$ events, with a jet faking a photon or hard radiation in a jet. The pure multi-jet no longer contributes to this final state, where we estimated a contamination probability of $<10^{-5}$. Given the small cross section for $p p>j j \gamma \gamma$, it is important to carefully estimate the contributions from $p p>j j j \gamma$. The right plot of figure 7 gives the purity fraction for the jjaa background from our simulation as a function of the $p_{T}$ of the subleading photon. Similarly to $j a$ purity in figure 6 , it exhibits a plateau at intermediate $p_{T}$ regime. However, it also exhibits a gradual decline with increasing $p_{T}$ : this can be attributed to the fact that the photons in $p p>j j \gamma \gamma$ events are mainly due to radiation and are not necessarily characterised by high $p_{T}$, while a high $p_{T}$ jet faking a photon is more probable for $p p>j j j \gamma$. This implies that both backgrounds have to be considered for the signal corresponding to the jjaa final state. 


\begin{tabular}{|l|c|c|c|}
\hline & $j j j j$ & $j j j a$ & $j j a a$ \\
\hline final state & $N_{j} \geq 4$ & $N_{j} \geq 3, \quad N_{a}=1$ & $N_{j} \geq 2, \quad N_{a}=2$ \\
\hline$p_{T}$ cuts & $p_{T}^{j}>80 \mathrm{GeV}$ & $p_{T}^{j}>150 \mathrm{GeV}$, & $p_{T}^{a}>50 \mathrm{GeV}$ \\
\hline pairs id. & \multicolumn{2}{|c|}{ min. $\sum_{i=\text { pairs }}\left|\Delta R_{i}-0.8\right|$} & min. $\Delta R_{a_{1} j_{i}}$ \\
\hline asymmetry & $\frac{\mid m_{j j_{1}-m_{j j_{2}} \mid}<0.1}{m_{j j_{1}+m_{j j_{2}}}<0}$ & $\frac{\left|m_{j a}-m_{j j}\right|}{m_{j a}+m_{j j}}<0.1$ & \\
parameters & $\left|\eta_{j j_{1}}-\eta_{j j_{2}}\right|<0.1$ & $\left|\eta_{j a}-\eta_{j j}\right|<0.1$ & \\
\hline binning & none & $m_{j a}$ & $m_{j a_{1}}$ \\
\hline
\end{tabular}

Table 3. Cut flow for the 3 final states, where the multi-jet one follows closely the CMS pair di-jet search of [21].

\subsection{Signal and background acceptances}

With an understanding of the different backgrounds, we proceed to estimating background and signal efficiencies corresponding to the event selection requirements. For the signal, we consider the following benchmark points, expressed in terms of masses and $14 \mathrm{TeV}$ pair production cross section, $\left(M_{\Phi}[\mathrm{GeV}], \sigma_{\text {pair }}[\mathrm{fb}]\right)$ :

$$
\text { BP1 :: }(900,74.2), \quad \text { BP2 :: }(1000,34.4), \quad \text { BP3 :: }(1100,16.7), \quad \text { BP4 :: }(1200,8.3) \text {. }
$$

These points are not yet excluded by the current searches, as shown in the previous section, in particular by the pair-dijet resonance search. To validate our analysis, we also consider a point at the edge of the excluded mass range:

$$
\text { BP0 :: }(700,400) \text {. }
$$

Like for the background, the parton level signal events are simulated at $14 \mathrm{TeV}$ using MADGRAPH [40] and showered by PYTHIA 8 [37]. We use the CMS card for DELPHES 3 [38] for the detector simulation. The jets are reconstructed using FASTJET [40], following the anti-kt algorithm with $R=0.4$ and $p_{T}=20 \mathrm{GeV}$.

First, for the multi-jet final state, we closely follow the CMS pair-dijet search of [21] by means of the cuts outlined in the second column of table 3 . We select events with at least 4 jets with $p_{T}>80 \mathrm{GeV}$. In order to select the two best di-jet pairs compatible with the signal, the four leading jets, ordered in $p_{T}$, are combined to create three unique combinations of di-jet pairs per event. Out of the three combinations, the di-jet configuration with the smallest $\Delta R_{\text {dijet }}=\sum_{i=1,2}\left|\Delta R_{i}-0.8\right|$ is chosen, where $\Delta R^{i}$ is the distance in the $\eta-\phi$ plane between the two jets in the $i^{\text {th }}$ di-jet pair. Once the best pairing is selected, two asymmetry parameters are defined, as in table 3, to reduce the QCD background, where $m_{j j_{i}}$ and $\eta_{j j_{i}}$ are the invariant mass and total pseudo-rapidity of the $i^{\text {th }}$ jet pair. To provide realistic estimates of the sensitivities, as the multi-jet background is hardly modelled by MonteCarlo generators, we decided to use instead the data-driven estimates used in the CMS search, shown in figure 9 of [21] for an accumulated luminosity of $35 \mathrm{fb}^{-1}$.

For the final state $j j j a$, given the absence of resonance searches, we adopt a methodology similar to the multi-jet searches: events are selected with one isolated photon with 


\begin{tabular}{|c|c|c|c|c|}
\hline & $j j j j$ & $j j j a$ & \multicolumn{2}{|c|}{$j j a a$} \\
\hline Signal acceptance $\epsilon_{S}\left(M_{\Phi}=1 \mathrm{TeV}\right)$ & 0.008 & 0.008 & \multicolumn{2}{|c|}{0.67} \\
\hline Background acceptance $\epsilon_{B}$ & Data & 0.002 & 0.69 & 0.009 \\
\hline Background cross section $(\mathrm{fb})$ & Data & $1.34 \times 10^{3}$ & 7 & $1.34 \times 10^{3}$ \\
\hline
\end{tabular}

Table 4. Acceptances for signal and backgrounds for the three final states we study, after the cuts in table 3. The signal corresponds to BP2, but the acceptances are rather independent on the mass value, while the background cross sections ae calculated after imposing a cut on the scalar sum of the $p_{T}$ of the outgoing particles $p_{T, \text { sum }}>1500 \mathrm{GeV}$. For $j j a a$, we show separately for the physical background $j j \gamma \gamma$ and the one from $j j j \gamma$ via jet mistagging.

$p_{T}>50 \mathrm{GeV}$ and at least 3 jets with $p_{T}>150 \mathrm{GeV}$. The best pairing of the photon with a jet is selected by use of the same technique as above, and furthermore we employ the $j j j j$ asymmetry variables with one $j j$ pair replaced by the $j a$ one. This approach was particularly useful in limiting the multi-jet QCD background, which we found to be significant in the jjja channel as shown in the purity plot of figure 7 . The cut-flow is summarized in the third column of table 3. Finally, in order to extract the sensitivities, we bin the events in the $m_{j a}$ distribution following the best pairing method described above. In table 4 we show the acceptance on signal and background after the cuts based on the asymmetry parameters, defined as the ratio of events that pass the cuts over the total number of generated events. In this instance the number for the both the signal and background indicates events which pass the selection due to the asymmetry parameters. We see that the signal acceptance for the multi-jet and jjja final states are similar.

The final state $j j a a$ is relatively simpler as there are only two combinatorial possibilities for the invariant mass reconstruction. Furthermore, the requirement of two isolated photons is sufficient to limit the multi-jet background to an insignificant amount. This makes it possible to adopt simpler selection criteria than the ones defined for multi-jet final state, and it is possible to increase the acceptance for signal events. We thus select events with at least two jets and exactly two isolated photons: this selection alone leads to the acceptances listed in the last column of table 4 . For the backgrounds, we listed separately the one coming from events with two real photons, and the one from $j j j \gamma$ : the latter is highly suppressed due to the low fake rate. Because of the natural suppression of the QCD background, there is no need to impose the cuts from the asymmetry parameters. ${ }^{6}$ We, therefore, content ourselves with identifying the correct pairing of photons and jets: after ordering the photons in $p_{T}$, we calculate the angular distance from the two jets and select the jet with the minimal value. The effectiveness of this strategy is shown in figure 8 , where we show the invariant masses of the two pairs for BP1 $\left(M_{\Phi}=900 \mathrm{GeV}\right)$ and BP4 $\left(M_{\Phi}=1200 \mathrm{GeV}\right)$. As it can be seen, both distributions nicely peak on the physical mass of $\Phi$.

\footnotetext{
${ }^{6}$ In principle, it would be possible to increase the significance by adding cuts on the asymmetry parameters, however this would reduce too much the background events in our simulation.
} 

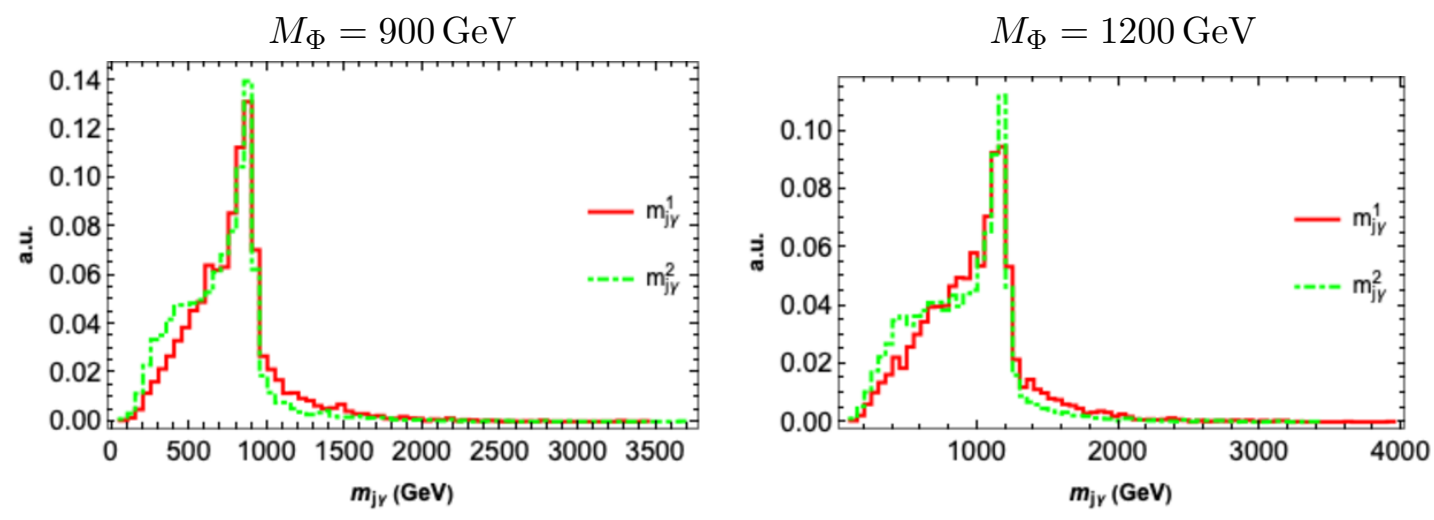

Figure 8. Distribution of the invariant masses $m_{j \gamma}$ of the two pairs for $M_{\Phi}=900 \mathrm{GeV}$ (left) and $1200 \mathrm{GeV}$ (right). See text for description.

\begin{tabular}{|c|c|c|c|}
\hline & $4 j$ & $3 j+1 \gamma$ & $2 j+2 \gamma$ \\
\hline$Z$ & 2.2 & $1.1(3.23)$ & $8.49(9.84)$ \\
\hline
\end{tabular}

Table 5. Comparison of signal sensitivities for the BP0 between different channels. The numbers are quoted for $35 \mathrm{fb}^{-1}$ of integrated luminosity. The number in bracket for the photon mode corresponds to bin-wise estimation of the signal sensitivity. See text for descriptions.

\subsection{Signal sensitivities}

With an estimation of the collider efficiencies for the signal and the background, we are in a position to compute the respective signal sensitivity. It must be pointed here that the acceptances for signal and backgrounds in table 4 are calculated naively using the events which satisfy the corresponding signal selection criteria. Since the mass of the underlying resonance is an unknown parameter, it is beneficial to define a variable sensitive to local variations in event multiplicities without biasing oneself to restricted regions of signal phase space. This can be put into practice by the definition of the following binned sensitivity [41]:

$$
Z^{\text {bin }}=\sqrt{\sum_{i}\left(2\left(s_{i}+b_{i}\right) \log \left[1+\frac{s_{i}}{b_{i}}\right]-2 s_{i}\right)},
$$

where $i$ runs over the bins and computes the signal and background events for a given observable in each bin. This facilitates a comparison of the signal and background events in each bin and is sensitive to the presence of signal events reconstructed away from the pole mass. This takes into account signal events which could have ordinarily been missed due to mass selection around the pole. Moreover it offers a fairly democratic search strategy as the mass of the underlying resonance is unknown. Note that, since the variable $Z^{\text {bin }}$ is a bin wise comparison, the bins which do not contain signal events do not contribute to the sensitivity and hence do not affect its computation.

To compare the sensitivity of the final states with photons to the multi-jet one, we make use of the ratios defined in eq. (3.1), where the sensitivity in the jjja and jjaa channels are computed using the binned formula of eq. (3.2). For the multi-jet final state, jjjj, 


\begin{tabular}{|c|c|c|c|c|c|}
\hline \multicolumn{3}{|c|}{ BP1 $(900,74.2)$} & \multicolumn{3}{|c|}{ BP3 $(1100,16.7)$} \\
\hline & $Y_{\chi}=1 / 3$ & $Y_{\chi}=2 / 3$ & \multicolumn{3}{|c|}{$Y_{\chi}=1 / 3$} \\
\hline$Z_{g g g g}$ & 10.32 & 7.48 & $Z_{g g g g}$ & 4.8 & 3.54 \\
\hline$Z_{g g g \gamma}$ & 5.02 & 13.03 & $Z_{g g g \gamma}$ & 1.09 & 3.06 \\
\hline$\delta_{g g g \gamma}$ & 0.48 & 1.74 & $\delta_{g g g \gamma}$ & 0.22 & 0.86 \\
\hline$Z_{g g \gamma \gamma}$ & 1.91 & 21.08 & $Z_{g g \gamma \gamma}$ & 0.47 & 5.31 \\
\hline$\delta_{g g \gamma \gamma}$ & 0.18 & 2.81 & $\delta_{g g \gamma \gamma}$ & 0.09 & 1.5 \\
\hline \multicolumn{3}{|c|}{ BP2 $(1000,34.4)$} & \multicolumn{3}{|c|}{ BP4 $(1200,8.3)$} \\
\hline & $Y_{\chi}=1 / 3$ & $Y_{\chi}=2 / 3$ & \multicolumn{2}{|c|}{$Y_{\chi}=1$} & $Y_{\chi}=2 / 3$ \\
\hline$Z_{g g g g}$ & 7.5 & 5.45 & $Z_{g g g g}$ & 2.6 & 1.91 \\
\hline$Z_{g g g \gamma}$ & 2.19 & 6.07 & $Z_{g g g \gamma}$ & 0.63 & 1.77 \\
\hline$\delta_{g g g \gamma}$ & 0.29 & 1.11 & $\delta_{g g g \gamma}$ & 0.24 & 0.92 \\
\hline$Z_{g g \gamma \gamma}$ & 0.98 & 10.97 & $Z_{g g \gamma \gamma}$ & 0.25 & 2.83 \\
\hline$\delta_{g g \gamma \gamma}$ & 0.13 & 2.01 & $\delta_{g g \gamma \gamma}$ & 0.09 & 1.48 \\
\hline
\end{tabular}

Table 6. Estimation of signal sensitivities $(Z)$ and ratio $\delta$ for different channels at HL-LHC with $3 \mathrm{ab}^{-1}$ of integrated luminosity. The multi-jet background at this luminosity is obtained by scaling the events multiplicities from $35 \mathrm{fb}^{-1}$. The benchmark points are denoted by $\left(M_{\Phi}[\mathrm{GeV}], \sigma_{\text {prod }}[\mathrm{fb}]\right)$.

since we use the CMS data at $35 \mathrm{fb}^{-1}$, we simply use $Z_{g g g g}=S / \sqrt{B}$ as the background is not known for different bin sizes. To validate our method, we fist focus on the benchmark point $\mathrm{BP} 0$, with branching ratios corresponding to the benchmark model with $Y_{\chi}=2 / 3$ (see table 1 ), for which $\operatorname{BR}(\Phi \rightarrow g g) \approx 80 \%$ and $\operatorname{BR}(\Phi \rightarrow g \gamma) \approx 15 \%$. The signal sensitivities for the different channels are given in table 5 for an integrated luminosity of $35 \mathrm{fb}^{-1}$, corresponding to the current CMS di-jet pair search. We first calculate the sensitivities using $Z=S / \sqrt{B}$, counting events in a mass window around the resonance, in a similar way for the three final states. Then, we indicate with numbers in brackets the results obtained with the binned sensitivity of eq. (3.2), where the bin size is $100 \mathrm{GeV}$, and the distributions correspond to $m_{j \gamma}$ for $j j j a$ and $m_{j \gamma_{1}}$ for $j j a a$ (invariant mass of the pair containing the highest $p_{T}$ photon). We also tested the stability against the bin size by varying it between 80 and $150 \mathrm{GeV}$ without noticing any significant variation. ${ }^{7}$ Our results show that, even without the binned sensitivity, the final state with two photons offers a much stronger reach compared to the multi-jet final state. Furthermore, the binning gives a significant increase in sensitivity, and we will use it in the following estimates.

We now turn our attention to the benchmark points BP1-BP4, with masses extended beyond the current exclusion. We first computed the expected sensitivities at the HL-LHC for an integrated luminosity of $3 \mathrm{ab}^{-1}$ for the benchmark models in table 1. For the multijet final state, we estimate the background by rescaling the ones from the CMS analysis at $35 \mathrm{fb}^{-1}$ to the higher luminosity. The results are shown in table 6 , where the bold

\footnotetext{
${ }^{7}$ Smaller bin sizes were not allowed for lack of statistics in the background events of our simulation.
} 

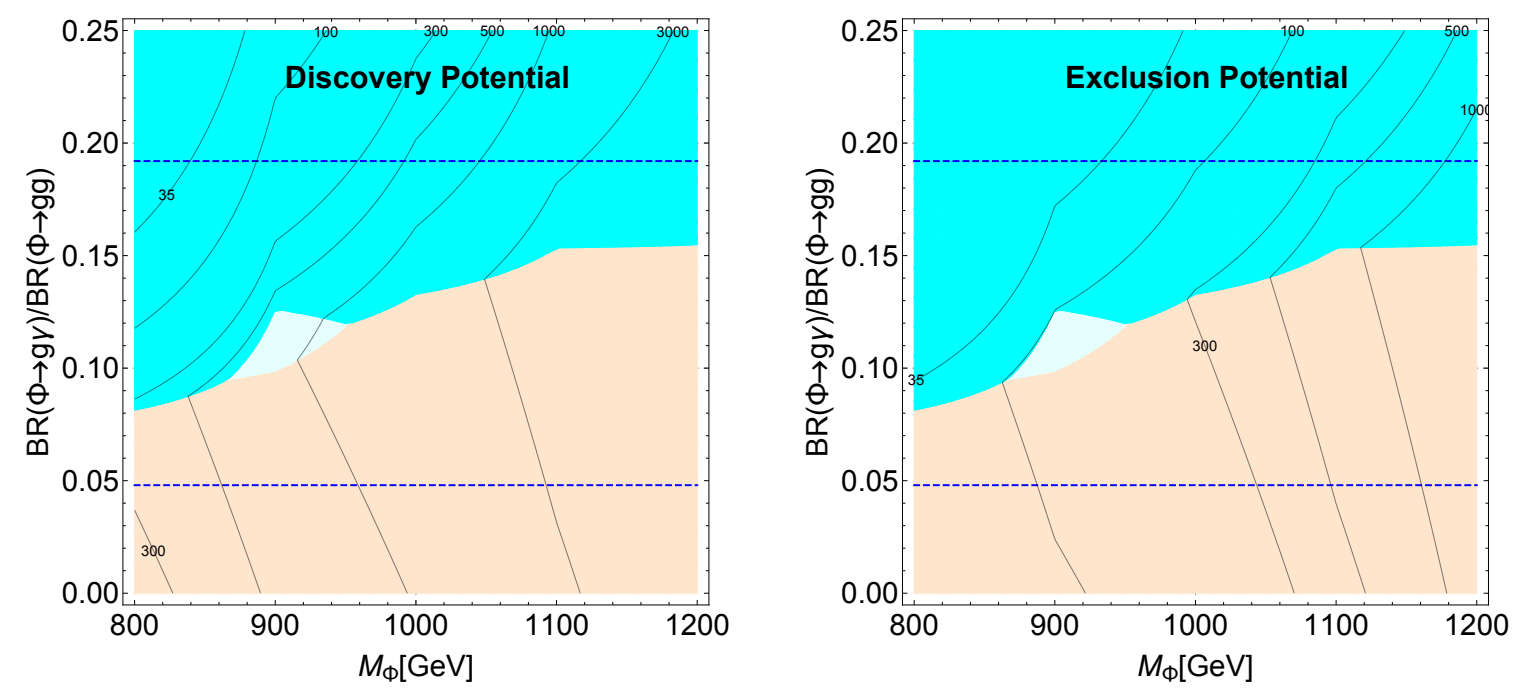

Figure 9. Contours of the luminosity with expected $Z=5$ ("Discovery Potential") on the left and $Z=2$ ("Exclusion Potential") on the right, as a function of $M_{\Phi}$ and the relative branching fraction $\mathcal{B}(\Phi \rightarrow g \gamma) / \mathcal{B}(\Phi \rightarrow g g)$, based on the HL-LHC analysis strategy in this subsection. The most sensitive channels are expected to be $g g \gamma \gamma$ (dark cyan), ggg (light cyan), or gggg (orange).

numbers correspond to the ratios defined in eq. (3.1). The values clearly show that, for the pessimistic case $Y_{\chi}=1 / 3$, the multi-jet final state always gives stronger reach having $\mathrm{BR}(\Phi \rightarrow g g) \approx 90 \%$. Instead, the optimistic case shows that it is the jjaa final state that provides the best sensitivity. In both cases, sensitivities close to $3 \sigma$ exclusion can be obtained, so that color octet masses up to $1.2 \mathrm{TeV}$ seem to be reachable at the HL-LHC (note that a combination of the three final states can further improve the reach).

The pattern of the ratios $\delta$ suggests that the relative dominance of a given mode depends on the relative branching fraction as well as the mass of the underlying resonance. This aspect is illustrated in figure 9 where we extend the analysis to generic ratios $B R(\Phi \rightarrow$ $g \gamma) / B R(\Phi \rightarrow g g)$. The two plots show contours of the integrated luminosity corresponding, on the left, to the "discovery potential" $(Z=5)$ and, on the right, to the "exclusion potential" $(Z=2)$, as a function of $M_{\Phi}$ and the relative branching fraction. In the dark cyan areas, the $g g \gamma \gamma$ channel has best projected sensitivity, while in the orange areas $g g g g$ is expected to dominate. The (small) light cyan area indicates best sensitivity of $g g g \gamma$ (which in this area is slightly stronger, but comparable to the other channels). The lighter masses exhibit a better reach of the $g g \gamma \gamma$ channel even for smaller values of the ratio $B R(\Phi \rightarrow g \gamma) / B R(\Phi \rightarrow g g)$. This can be attributed to the significant multi-jet background in the lower invariant mass bins. However, with increasing $M_{\phi}$ the background rate drops more rapidly than for the $g g \gamma \gamma$ channel (for which less severe cuts have been applied). The projection for heavier masses is limited on two accounts: lack of background statistics and rapidly dropping signal cross sections. The advent of future proton colliders would serve as a natural continuation to probe these states and will be discussed in the next section. 


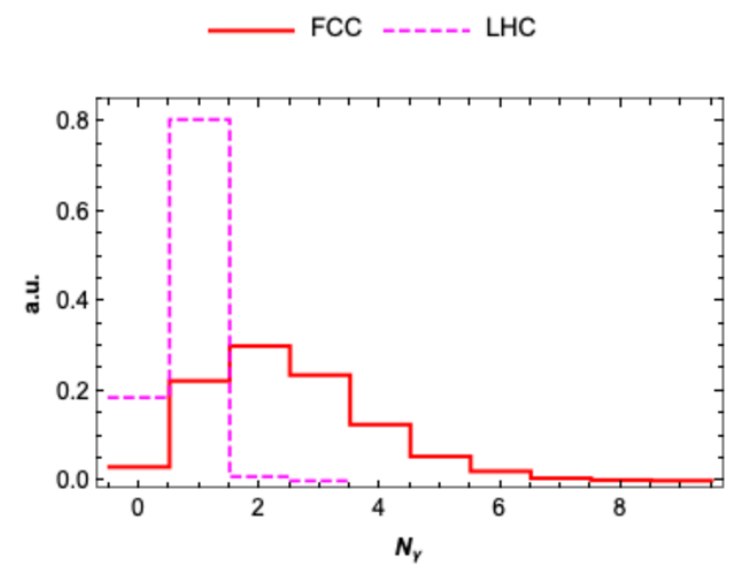

Figure 10. Comparison of photon multiplicities $N_{\gamma}$ for the $g g g a$ signal events between the HL-LHC and the FCC-hh.

\subsection{The next collider: FCC-hh}

The analysis in the previous sections shows that the HL-LHC is expected to have good sensitivity to probe masses $M_{\Phi} \leq 1.2 \mathrm{TeV}$, and that adding searches for final states with one and two photons can help in significantly extending the reach. This analysis offers a natural progression leading to the exploration of higher masses at a future hadronic collider, like the FCC-hh at a center-of-mass energy of $100 \mathrm{TeV}$. While the FCC can be expected to probe masses as heavy as tens of $\mathrm{TeV}$, as a first test we estimated the reach for the following two benchmark points, $\left(M_{\Phi}[\mathrm{TeV}], \sigma_{\text {pair }}[\mathrm{fb}]\right)$ :

$$
\text { BP5 :: }(2.5,270), \quad \text { BP6 :: }(3,100) \text {; }
$$

where the cross sections correspond to the FCC-hh energies. We simulated both signal and backgrounds using the standard FCC-hh cards, where the background samples in this phase space is generated by requiring the scalar $p_{T}$ sum of the outgoing particles to be atleast $2 \mathrm{TeV}$. One key difference between the analyses at the two machines is the difference in the photon multiplicities. For the HL-LHC, the event selection was associated with the requirement of exactly one or two isolated photons, satisfying a certain minimum $p_{T}$ criterion that corresponds to the signal. However, given the larger center of mass energy at the FCC, the radiated photons are also expected to be both isolated and hard. The difference in photon multiplicities, after the cuts, between the two machines is illustrated in figure 10. As a result, the requirement of exactly one or two isolated photons would diminish the signal acceptance. In light of this the event selection is slightly modified with respect to the HL-LHC one, with the requirement of at-least a single isolated photon. However, the signal acceptance efficiencies after the cuts is machine independent and roughly mirrors the values given in table 4 .

There is also another key difference between the analyses at the two machines: the photon $p_{T}$ spectrum for the background. Since there is a larger probability for the radiated photons to pass the isolation criteria at the FCC, it is also instructive to compare its $p_{T}$ 

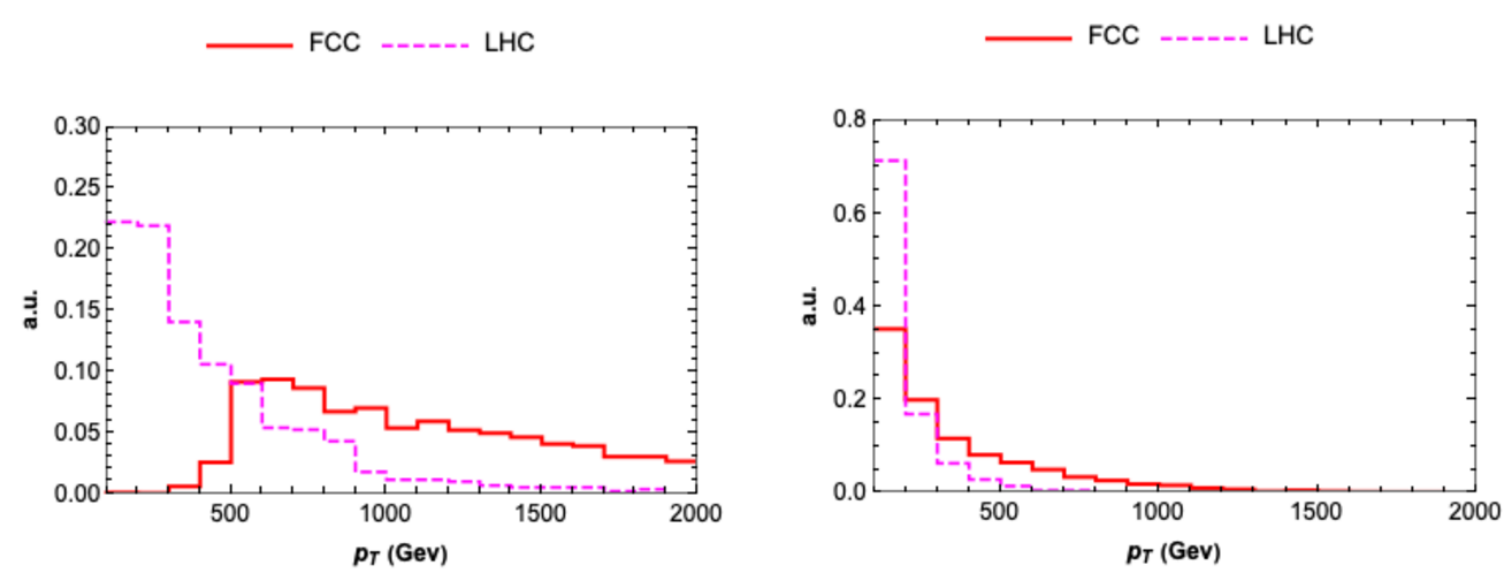

Figure 11. Comparison of $p_{T}$ distribution for the $j$ jja background between the HL-LHC and the FCC-hh. The left plot is for the leading $p_{T}$ photon and the right plot is for the sub-leading one.

\begin{tabular}{|ccc|ccc|}
\hline \multicolumn{3}{|c|}{ BP1 (2.5,270) } & \multicolumn{3}{c|}{ BP2 (3,100) } \\
& $Y_{\chi}=1 / 3$ & $Y_{\chi}=2 / 3$ & & $Y_{\chi}=1 / 3$ & $Y_{\chi}=2 / 3$ \\
\hline$Z_{g g g \gamma}$ & 11.7 & 30.8 & $Z_{g g g \gamma}$ & 4.98 & 13.6 \\
\hline$Z_{g g \gamma \gamma}$ & 5.47 & 58.2 & $Z_{g g \gamma \gamma}$ & 2.87 & 31.1 \\
\hline
\end{tabular}

Table 7. Estimation of signal sensitivities $(Z)$ for different channels at $3 \mathrm{ab}^{-1}$ of integrated luminosity for the FCC. We recall that the numbers for the $j j j \gamma$ signal is underestimated as the mulit-jet background has not been considered in its evaluation.

distribution with respect to the HL-LHC. For illustration we consider the $j j j \gamma$ background, and compare the $p_{T}$ of the leading and the sub-leading $p_{T}$ photons at the two colliders. Figure 11 illustrates this difference for the leading photon (left) and the sub-leading photon (right). Note the significantly longer tail at the FCC, which eventually leads to the presence of background in larger invariant mass bins. This suggests that for the $j j a a$, the $j j j \gamma$ background is more relevant at the FCC. The same argument can be also be extended to the relevance of the multi-jet background for the signal with one photon and three jets. Given the computational limitations, we cannot accurately represent the multi-jet backgrounds, thus we do not consider them in our estimates for computing the signal acceptance. As a result the numbers for the jjja final state are likely to be overestimated. Nevertheless, since the multi-jet background is unlikely to generate two hard photons, our estimates for the jja final state $\left(p_{T}^{\gamma}>150 \mathrm{GeV}\right)$ are likely to be accurate. The corresponding values of sensitivities are given in table 7 , we use mass bins of width $350 \mathrm{GeV}$ to avoid fluctuations in background statistics. Our results clearly show that the FCC-hh will have an excellent reach for a color octet by looking at final states with photons. 


\section{Conclusion}

Heavy colored composite states are a feature of several frameworks beyond the SM. A coupling to the gluons, proportional to $\alpha_{s}$, ensures that their decay into the $g g$ final state is the most dominant mode. This would naturally motivate searches for these states in the multi-jet final state. In this work we invoke the possibility of the coupling of these states to a gluon and a photon through the WZW term. In the pair production of these states, we show that final states with two photons (and to a minor extent with one photon) could be more sensitive than the multi-jet final state. Depending on the underlying mass and the ratio $B R(\Phi \rightarrow g \gamma) / B R(\Phi \rightarrow g g)$, we identify the most dominant mode for their discovery (or for setting stronger exclusions). The reach for the HL-LHC extends up to $1.2 \mathrm{TeV}$. Masses beyond this range are better accessed at a FCC, and we provide preliminary estimates for a center-of-mass energy of $100 \mathrm{TeV}$. This study does not only strongly motivate the exploration of pair produced color octets with one and two photons, but also does offer a natural progression from the LHC to future proton colliders.

\section{Acknowledgments}

We wish to thank V. Sordini for useful comments and discussions, and comments on the manuscript. AI, GC, AD would like to thank CEFIPRA Indo-French research network. GC and AD acknowledge partial support from the France-Korea Particle Physics Lab (FKPPL) and the Labex-LIO (Lyon Institute of Origins) under grant ANR-10-LABX-66 (Agence Nationale pour la Recherche), and FRAMA (FR3127, Fédération de Recherche "André Marie Ampère"). TF's work is supported by IBS under the project code IBSR018-D1. GC, AD and TF would like to express a special thanks to the Mainz Institute for Theoretical Physics (MITP) of the Cluster of Excellence PRISMA+ (Project ID 39083149) for its hospitality and support during part of this work.

\section{A Bookkeeping of neutral color octet models}

In this appendix we present a classification of models that contain a color octet pseudoscalar. The key ingredient is to add information about the electroweak quantum numbers of the multiplet the neutral state belongs to. We will classify the cases in terms of the weak isospin $I, \Phi_{I}$, with $I=1,2,3$ as minimal cases, and focus on the neutral pseudo-scalar components matching the effective Lagrangian in eq. (1.1).

Isospin 1. In this case, the multiplet has a single neutral component, $\Phi_{1}$. The leading order couplings to tops and gauge bosons arise at dim- 5 level, and can be written in a gauge-invariant way as

$$
\mathcal{L}_{\Phi_{1}}=i \Phi_{1}^{a}\left(\frac{\lambda_{t}}{f_{\Phi}} \varphi_{H}^{\dagger} \bar{q}_{L} \frac{\lambda^{a}}{2} t_{R}-\text { h.c. }\right)+\frac{\alpha_{s} \kappa_{g}}{8 \pi f_{\Phi}} \Phi_{1}^{a} \epsilon^{\mu \nu \rho \sigma}\left[\frac{1}{2} d^{a b c} G_{\mu \nu}^{b} G_{\rho \sigma}^{c}+\frac{g^{\prime} \kappa_{B}}{g_{s} \kappa_{g}} G_{\mu \nu}^{a} B_{\rho \sigma}\right]
$$


where $\varphi_{H}$ is the Higgs doublet and $\mathrm{SU}(2)_{L}$ contractions are left understood. After the Higgs field develops a vacuum expectation value $\left\langle\varphi_{H}^{\dagger} \varphi_{H}\right\rangle=v^{2} / 2$, we have the following effective couplings

$$
C_{t}=\frac{\lambda_{t} v}{\sqrt{2} m_{t}}, \quad \kappa_{\gamma}=\kappa_{Z}=\kappa_{B} .
$$

This case occurs in models of top partial compositeness with an underlying gauge-fermion description $[2,6]$.

Isospin 2. In this case, the neutral color octet belongs to a doublet with hypercharge $Y_{\Phi}=1 / 2$, which also contains a charged component and a neutral scalar:

$$
\Phi_{2}=\left(\begin{array}{c}
\Phi_{2}^{+} \\
\left(\tilde{\Phi}_{2,0}+i \Phi_{2,0}\right) / \sqrt{2}
\end{array}\right) .
$$

This case is interesting as a scalar extension of the SM [42], as it does not suffer from large flavour changing neutral currents [43]. Now, it is possible to write a dim-4 coupling to tops:

$$
\mathcal{L}_{\Phi_{2}} \supset \lambda_{t}\left(\Phi_{2}^{\dagger}\right)^{a} \bar{q}_{L} \frac{\lambda^{a}}{2} t_{R}+\text { h.c. }
$$

which gives a large $C_{t}=\frac{\lambda_{t} f_{\Phi}}{\sqrt{2} m_{t}}$, plus similar couplings of the real and charged components. Thus, if the coupling to tops is present, decays will be dominated by $t \bar{t}$.

Couplings to gauge bosons can only arise at dim- 6 , in the form:

$$
\begin{aligned}
\mathcal{L}_{\Phi_{2}} \supset & \frac{g_{s}^{2} c_{g}}{32 \pi^{2} f_{\Phi}^{2}}\left(i \varphi_{H}^{\dagger} \Phi_{2}^{a}+\text { h.c. }\right) \epsilon^{\mu \nu \rho \sigma} \frac{1}{2} d^{a b c} G_{\mu \nu}^{b} G_{\rho \sigma}^{c} \\
& +\frac{g_{s} g^{\prime} c_{B}}{32 \pi^{2} f_{\Phi}^{2}}\left(i \varphi_{H}^{\dagger} \Phi_{2}^{a}+\text { h.c. }\right) \epsilon^{\mu \nu \rho \sigma} G_{\mu \nu}^{a} B_{\rho \sigma} \\
& +\frac{g_{s} g c_{W}}{32 \pi^{2} f_{\Phi}^{2}}\left(i \varphi_{H}^{\dagger} \tau^{\alpha} \Phi_{2}^{a}+\text { h.c. }\right) \epsilon^{\mu \nu \rho \sigma} G_{\mu \nu}^{a} W_{\rho \sigma}^{\alpha}
\end{aligned}
$$

Matching leads to the following effective couplings:

$$
\kappa_{g}=c_{g} \frac{v}{f_{\Phi}}, \quad \kappa_{\gamma}=\left(c_{B}+c_{W}\right) \frac{v}{2 f_{\Phi}}, \quad \kappa_{Z}=\left(c_{B}-\frac{c_{W}}{\tan ^{2} \theta_{W}}\right) \frac{v}{2 f_{\Phi}} .
$$

Isospin 3. The minimal choice is to embed the neutral pseudo-scalar in an isotriplet with zero hypercharge, together with charged components:

$$
\Phi_{3}=\left(\begin{array}{c}
\Phi_{3}^{+} \\
\Phi_{3,0} \\
\Phi_{3}^{-}
\end{array}\right)
$$

The leading couplings arise at dim-5 in the form:

$$
\mathcal{L}_{\Phi_{3}}=i \Phi_{3}^{a \alpha}\left(\frac{\lambda_{t}}{f_{\Phi}}\left(\varphi_{H}^{\dagger} \tau^{\alpha} \bar{q}_{L}\right) \frac{\lambda^{a}}{2} t_{R}-\text { h.c. }\right)+\frac{g_{s} g \kappa_{W}}{32 \pi^{2} f_{\Phi}} \Phi_{3}^{a \alpha} \epsilon^{\mu \nu \rho \sigma} G_{\mu \nu}^{a} W_{\rho \sigma}^{\alpha} .
$$



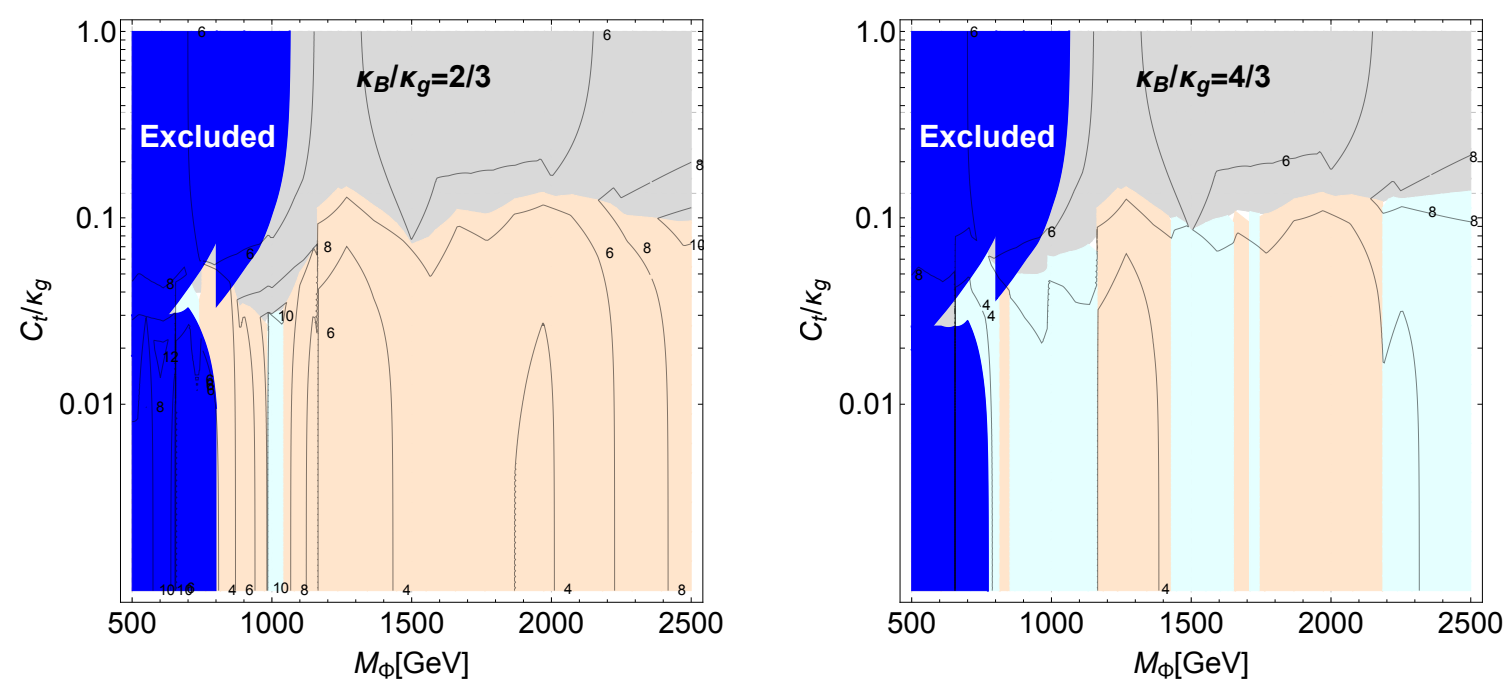

Figure 12. Observed bounds on color octet single production in the $M_{\Phi}$ vs. $C_{t} / \kappa_{g}$ plane for $\kappa_{B} / \kappa_{g}=2 / 3$ and $4 / 3$. The contours show the upper bound on $\kappa_{g} / f_{\Phi}\left(\right.$ in $\left.\mathrm{TeV}^{-1}\right)$. The dark-blue areas are excluded by pair production searches. In the grey areas, the strongest bound arises from $t \bar{t}$ resonance searches. In the orange (cyan) areas, the currently strongest bound arises from di-jet $($ jet- $\gamma)$ searches.

In this case, the matching leads to

$$
C_{t}=\frac{\lambda_{t} v}{2 \sqrt{2} m_{t}}, \quad \kappa_{\gamma}=\kappa_{W}, \quad \kappa_{Z}=-\frac{1}{\tan ^{2} \theta_{W}} \kappa_{W}
$$

Interestingly, in this case couplings to two gluons are suppressed by two Higgs insertions, i.e. they arise at dim-7 level with $\kappa_{g} \propto v^{2} / f_{\Phi}^{2}$. Thus, decays into $g \gamma$ and $g Z$ are dominant, with

$$
\frac{\operatorname{BR}\left(\Phi_{3,0} \rightarrow g Z\right)}{\operatorname{BR}\left(\Phi_{3,0} \rightarrow g \gamma\right)} \approx \tan ^{-2} \theta_{W} \approx 3.5
$$

thus dominant decays into $Z$ final states.

\section{B Current limits from single production}

In section 2.1, we have presented upper bounds on $\kappa_{g} / f_{\Phi}$ deriving from expected bounds from searches sensitive to single production. In this appendix we present similar plots, drawn from the observed bounds. Figure 12 corresponds to figure 4 showing results for the two benchmark models, while figure 13 corresponds to figure 5 in showing limits for $C_{t}=0$.

Comparing the two pairs of figures clearly highlights that the limits on the coupling $\kappa_{g} / f_{\Phi}$ are comparable, as well as the regions of dominance of each final state. The main difference is that the plots in this appendix are much more irregular, due to the statistical fluctuations in the observed bounds (C.f. figure 3). 


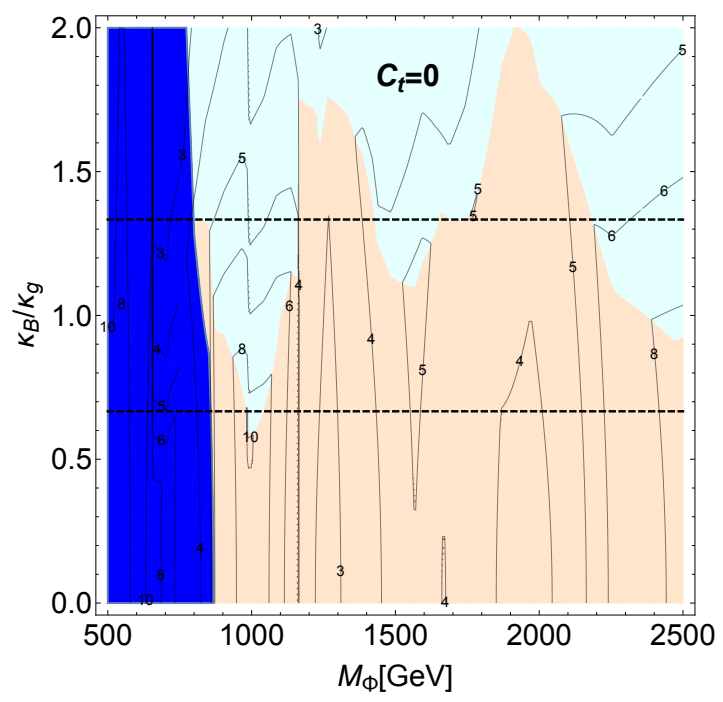

Figure 13. Observed bounds on color octet single production in the $M_{\Phi}$ vs. $\kappa_{B} / \kappa_{g}$ plane. The dark blue area is excluded from pair production bounds, while the orange (cyan) area labels where the dominant bound arises from the $j j(j \gamma)$ search. Contours show the upper bound on $\kappa_{g} / f_{\Phi}$ in $\mathrm{TeV}^{-1}$. For reference, the horizontal dashed lines indicate fixed $\kappa_{B} / \kappa_{g}$ ratios of $2 / 3$ (lower) and $4 / 3$ (higher).

Open Access. This article is distributed under the terms of the Creative Commons Attribution License (CC-BY 4.0), which permits any use, distribution and reproduction in any medium, provided the original author(s) and source are credited.

\section{References}

[1] C.-Y. Chen, A. Freitas, T. Han and K.S.M. Lee, Heavy color-octet particles at the LHC, JHEP 05 (2015) 135 [arXiv: 1410.8113] [INSPIRE].

[2] G. Cacciapaglia, H. Cai, A. Deandrea, T. Flacke, S.J. Lee and A. Parolini, Composite scalars at the LHC: the Higgs, the sextet and the octet, JHEP 11 (2015) 201 [arXiv:1507.02283] [INSPIRE].

[3] J. Barnard, T. Gherghetta and T.S. Ray, UV descriptions of composite Higgs models without elementary scalars, JHEP 02 (2014) 002 [arXiv: 1311.6562] [INSPIRE].

[4] G. Ferretti and D. Karateev, Fermionic UV completions of composite Higgs models, JHEP 03 (2014) 077 [arXiv: 1312.5330] [INSPIRE].

[5] C. Englert, G. Ferretti and M. Spannowsky, Jet-associated resonance spectroscopy, Eur. Phys. J. C 77 (2017) 842 [arXiv: 1706.04242] [InSPIRE].

[6] A. Belyaev et al., Di-boson signatures as standard candles for partial compositeness, JHEP 01 (2017) 094 [Erratum ibid. 12 (2017) 088] [arXiv: 1610.06591] [INSPIRE].

[7] F. Hayot and O. Napoly, Detecting a heavy colored object at the FNAL Tevatron, Z. Phys. C 7 (1981) 229 [INSPIRE].

[8] A. Belyaev, R. Rosenfeld and A.R. Zerwekh, Tevatron potential for technicolor search with prompt photons, Phys. Lett. B 462 (1999) 150 [hep-ph/9905468] [INSPIRE]. 
[9] ATLAS collaboration, Analysis of events with b-jets and a pair of leptons of the same charge in pp collisions at $\sqrt{s}=8 \mathrm{TeV}$ with the ATLAS detector, JHEP 10 (2015) 150 [arXiv: 1504.04605] [INSPIRE].

[10] ATLAS collaboration, Search for production of vector-like quark pairs and of four top quarks in the lepton-plus-jets final state in pp collisions at $\sqrt{s}=8 \mathrm{TeV}$ with the ATLAS detector, JHEP 08 (2015) 105 [arXiv: 1505.04306] [INSPIRE].

[11] CMS collaboration, Search for pair-produced resonances decaying to jet pairs in proton-proton collisions at $\sqrt{s}=8 \mathrm{TeV}$, Phys. Lett. B 747 (2015) 98 [arXiv:1412.7706] [INSPIRE].

[12] C. Degrande, B. Fuks, V. Hirschi, J. Proudom and H.-S. Shao, Automated next-to-leading order predictions for new physics at the LHC: the case of colored scalar pair production, Phys. Rev. D 91 (2015) 094005 [arXiv:1412.5589] [INSPIRE].

[13] L. Darmé, B. Fuks and M. Goodsell, Cornering sgluons with four-top-quark events, Phys. Lett. B 784 (2018) 223 [arXiv: 1805.10835] [INSPIRE].

[14] CMS collaboration, Search for Standard Model production of four top quarks with same-sign and multilepton final states in proton-proton collisions at $\sqrt{s}=13$ TeV, Eur. Phys. J. C 78 (2018) 140 [arXiv: 1710.10614] [INSPIRE].

[15] CMS collaboration, Search for production of four top quarks in final states with same-sign or multiple leptons in proton-proton collisions at $\sqrt{s}=13$ TeV, Eur. Phys. J. C 80 (2020) 75 [arXiv: 1908.06463] [INSPIRE].

[16] ATLAS collaboration, Search for pair production of up-type vector-like quarks and for four-top-quark events in final states with multiple b-jets with the ATLAS detector, JHEP 07 (2018) 089 [arXiv : 1803.09678] [INSPIRE].

[17] ATLAS collaboration, Search for new phenomena in events with same-charge leptons and b-jets in pp collisions at $\sqrt{s}=13 \mathrm{TeV}$ with the ATLAS detector, JHEP 12 (2018) 039 [arXiv: 1807.11883] [INSPIRE].

[18] ATLAS collaboration, Search for four-top-quark production in the single-lepton and opposite-sign dilepton final states in pp collisions at $\sqrt{s}=13 \mathrm{TeV}$ with the ATLAS detector, Phys. Rev. D 99 (2019) 052009 [arXiv: 1811.02305] [INSPIRE].

[19] CMS collaboration, Search for the production of four top quarks in the single-lepton and opposite-sign dilepton final states in proton-proton collisions at $\sqrt{s}=13 \mathrm{TeV}$, JHEP 11 (2019) 082 [arXiv: 1906.02805] [INSPIRE].

[20] ATLAS collaboration, A search for pair-produced resonances in four-jet final states at $\sqrt{s}=13 \mathrm{TeV}$ with the ATLAS detector, Eur. Phys. J. C 78 (2018) 250 [arXiv:1710.07171] [INSPIRE].

[21] CMS collaboration, Search for pair-produced resonances decaying to quark pairs in proton-proton collisions at $\sqrt{s}=13$ TeV, Phys. Rev. D 98 (2018) 112014 [arXiv: 1808.03124] [INSPIRE].

[22] B. Bhattacherjee, P. Byakti, A. Kushwaha and S.K. Vempati, Unification with vector-like fermions and signals at LHC, JHEP 05 (2018) 090 [arXiv:1702.06417] [INSPIRE].

[23] CMS collaboration, Search for resonant $t \bar{t}$ production in proton-proton collisions at $\sqrt{s}=13 \mathrm{TeV}$, JHEP 04 (2019) 031 [arXiv:1810.05905] [INSPIRE]. 
[24] ATLAS collaboration, Search for heavy particles decaying into top-quark pairs using lepton-plus-jets events in proton-proton collisions at $\sqrt{s}=13$ TeV with the ATLAS detector, Eur. Phys. J. C 78 (2018) 565 [arXiv: 1804.10823] [INSPIRE].

[25] ATLAS collaboration, Search for heavy particles decaying into a top-quark pair in the fully hadronic final state in pp collisions at $\sqrt{s}=13 \mathrm{TeV}$ with the ATLAS detector, Phys. Rev. D 99 (2019) 092004 [arXiv: 1902.10077] [INSPIRE].

[26] CMS collaboration, Search for narrow and broad dijet resonances in proton-proton collisions at $\sqrt{s}=13 \mathrm{TeV}$ and constraints on dark matter mediators and other new particles, JHEP 08 (2018) 130 [arXiv: 1806.00843] [INSPIRE].

[27] CMS collaboration, Searches for dijet resonances in pp collisions at $\sqrt{s}=13 \mathrm{TeV}$ using the 2016 and 2017 datasets, CMS-PAS-EXO-17-026, CERN, Geneva, Switzerland (2017).

[28] ATLAS collaboration, Search for new phenomena in dijet events using $37 \mathrm{fb}^{-1}$ of $\mathrm{pp}$ collision data collected at $\sqrt{s}=13$ TeV with the ATLAS detector, Phys. Rev. D 96 (2017) 052004 [arXiv: 1703.09127] [INSPIRE].

[29] ATLAS collaboration, Search for low-mass dijet resonances using trigger-level jets with the ATLAS detector in pp collisions at $\sqrt{s}=13$ TeV, Phys. Rev. Lett. 121 (2018) 081801 [arXiv: 1804.03496] [INSPIRE].

[30] ATLAS collaboration, Search for new resonances in mass distributions of jet pairs using $139 \mathrm{fb}^{-1}$ of pp collisions at $\sqrt{s}=13 \mathrm{TeV}$ with the ATLAS detector, JHEP 03 (2020) 145 [arXiv: 1910.08447] [INSPIRE].

[31] ATLAS collaboration, Search for new phenomena in high-mass final states with a photon and a jet from pp collisions at $\sqrt{s}=13 \mathrm{TeV}$ with the ATLAS detector, Eur. Phys. J. C 78 (2018) 102 [arXiv : 1709.10440] [INSPIRE].

[32] CMS collaboration, Search for excited quarks of light and heavy flavor in $\gamma+$ jet final states in proton-proton collisions at $\sqrt{s}=13 \mathrm{TeV}$, Phys. Lett. B 781 (2018) 390 [arXiv: 1711.04652] [INSPIRE].

[33] ATLAS collaboration, Search for new phenomena in photon + jet events collected in proton-proton collisions at $\sqrt{s}=8 \mathrm{TeV}$ with the ATLAS detector, Phys. Lett. B 728 (2014) 562 [arXiv: 1309.3230] [INSPIRE].

[34] CMS collaboration, Search for excited quarks in the $\gamma+$ jet final state in proton-proton collisions at $\sqrt{s}=8 \mathrm{TeV}$, Phys. Lett. B 738 (2014) 274 [arXiv:1406.5171] [InSPIRE].

[35] G. Cacciapaglia, A. Deandrea, T. Flacke and A.M. Iyer, Gluon-photon signatures for color octet at the LHC (and beyond), arXiv:2002.01474 [INSPIRE].

[36] N. Bizot, G. Cacciapaglia and T. Flacke, Common exotic decays of top partners, JHEP 06 (2018) 065 [arXiv:1803.00021] [INSPIRE].

[37] T. Sjöstrand, S. Mrenna and P.Z. Skands, A brief introduction to PYTHIA 8.1, Comput. Phys. Commun. 178 (2008) 852 [arXiv:0710.3820] [INSPIRE].

[38] DELPHES 3 collaboration, DELPHES 3, a modular framework for fast simulation of a generic collider experiment, JHEP 02 (2014) 057 [arXiv: 1307.6346] [INSPIRE].

[39] CMS collaboration, Measurement of triple-differential cross section of gamma + jet production, CMS-PAS-QCD-11-005, CERN, Geneva, Switzerland (2013). 
[40] J. Alwall et al., The automated computation of tree-level and next-to-leading order differential cross sections and their matching to parton shower simulations, JHEP 07 (2014) 079 [arXiv: 1405.0301] [INSPIRE].

[41] G. Cowan, K. Cranmer, E. Gross and O. Vitells, Asymptotic formulae for likelihood-based tests of new physics, Eur. Phys. J. C 71 (2011) 1554 [Erratum ibid. C 73 (2013) 2501] [arXiv: 1007.1727] [INSPIRE].

[42] A.V. Manohar and M.B. Wise, Flavor changing neutral currents, an extended scalar sector and the Higgs production rate at the CERN LHC, Phys. Rev. D 74 (2006) 035009 [hep-ph/0606172] [INSPIRE].

[43] J.M. Arnold, M. Pospelov, M. Trott and M.B. Wise, Scalar representations and minimal flavor violation, JHEP 01 (2010) 073 [arXiv:0911.2225] [INSPIRE]. 